\title{
Plankton accumulation and transport in propagating nonlinear internal fronts
}

\author{
by A. Scotti ${ }^{1}$ and J. Pineda ${ }^{2}$
}

\begin{abstract}
Accumulation and transport of plankton in fronts propagating across-shore is a process of considerable ecological importance for many inhabitants of the littoral zone, since it links the offshore larval pool with the juvenile and adult inshore habitat. Several field studies have shown that larval plankton accumulates in fronts, but have failed to give a conclusive proof that effective Lagrangian transport takes place. A few process-oriented numerical studies have lent support to the idea, but the scope of their results is limited by the two-dimensional nature of the flows considered and by the simple model used to account for the behavior of plankton. In this paper, we relax both constraints. We solve the three-dimensional Navier-Stokes equation to compute the time dependent velocity field, and we use an empirically based model for the behavior of plankton. Our results show that accumulation and transport is possible, even for larvae characterized by sustained swimming speeds that are small compared with the speed of propagation of the front. We introduce a simple model to characterize the accumulation along the front, which includes both entrainment and detrainment. The model accurately represents accumulation calculated from the numerical runs, and provide a simple tool to estimate transport under a variety of circumstances. We also investigate the spatial distribution of plankton along and across the front and show that it is very patchy and dependent on the swimming speed of plankton, with important implications for interpreting results from field experiments.
\end{abstract}

\section{Introduction}

Many inhabitants of the littoral zone with a planktonic larval stage face the problem of reaching the shoreline in order to settle and reproduce. For example, larvae of littoral barnacles, after spending the larval stage offshore, need to reach a suitable rocky substrate in the intertidal region to settle and metamorph into adult organisms. However, while alongshore transport processes are usually quite energetic because of the existence of nonzero mean Eulerian currents, across-shore processes are much less effective, since the Eulerian mean is zero or very small (as when upwelling/downwelling is present). Hence, from an ecological point of view, it is important to recognize possible mechanisms of cross-shore transport. At large scales, the horizontal migration of buoyant plumes (such as coastally

1. Department of Marine Sciences, University of North Carolina, Chapel Hill, North Carolina, 27599, U.S.A.email: ascotti@unc.edu

2. Department of Biology, Woods Hole Oceanographic Institution, Woods Hole, Massachusetts, 02543, U.S.A. 
trapped river plumes) in response to wind events has been shown to transfer plankton from an offshore pool and move it closer to shore (Hetland et al., 2003). However, this mechanism is limited to areas where large-scale buoyant plumes occur (such as near estuaries) and does not solve the problem of reaching the near-shore area. Norris (1963) first postulated that surface slicks over internal waves could transport larvae onshore. The idea was later picked-up by Shanks and coworkers (Shanks, 1982, 1983, 1985, 1987, 1988; Shanks and Wright, 1987). The hypothesis rested on the well known fact that oil slicks often form near the surface convergence zone generated by the waves (Ewing, 1954). Shanks and Wright (1987) found that barnacle cyprids were more abundant over the convergence zone of internal waves and concluded that internal waves do in fact transport plankton. Lennert-Cody and Franks (1999) modeled how the concentration of actively swimming plankton is spatially modulated by the passage of linear and nonlinear internal waves. The plankton was assumed to swim vertically and against the current, with a speed assumed to be a taxon-dependent fraction of the fluid speed, and an advection/diffusion equation was solved for the Eulerian concentration. When applied to weakly nonlinear waves, this model shows increase in the concentration. However, the observation of increased concentration in the Eulerian frame of reference per se is not sufficient to conclude that transport occurs. In fact, it is necessary to show that the same group of larvae is transported onshore in the Lagrangian sense, with a speed comparable to the celerity of the wave. Using a fully nonlinear two-dimensional model Lamb (1997) showed that weakly-nonlinear waves are not very effective in transporting plankton, unless larvae actively swim in the horizontal direction with speeds approaching the celerity of the wave. The same simulations hinted that strongly nonlinear waves can do what weakly-nonlinear waves cannot. Observations suggest that weakly-nonlinear waves are the most common form of nonlinear internal waves on the shelf (Jackson, 2004). On the other hand, a mounting body of evidence suggests that strongly nonlinear waves might be more prevalent in nearshore areas (Pineda, 1999; Leichter et al., 2003; Klymak and Moum, 2003; Scotti and Pineda, 2004; Hosegood and van Haren, 2004). Here, strong nonlinearity refers to the appearance of regions of closed streamlines when viewed from a frame of reference moving with the waves. In the observations, they fall into two categories: gravity currents (Pineda, 1991, 1999; Leichter et al., 2003; Hosegood and van Haren, 2004) if the advancing front remains connected with an offshore reservoir, or nonlinear waves with trapped cores (also known as detached gravity currents) (Manasseh et al., 1998; Klymak and Moum, 2003; Scotti and Pineda, 2004), if the front detaches from the reservoir. In reality, the same event may start as a gravity current and evolve into a detached gravity current as the front propagates. Most laboratory studies consider gravity currents, detached or not, propagating on a solid boundary, where the usual no-slip boundary condition applies (Manasseh et al., 1998; Simpson, 1999). This same case has been studied with fully threedimensional computations (Härtel et al., 2000a,b). In this case, the head is unstable due to lobe-and-cleft instabilities, which leads to different mixing properties near the head relative to a current propagating along a free-slip surface. The case of a front propagating along the surface of a fluid, where we expect free-slip conditions, has been considered numerically 
only with two-dimensional models (Härtel et al., 2000a; Helfrich and Pineda, 2003). At lab scales, impurities accumulating on the surface conjure with surface tension to replace the free-slip condition with an effective no-slip condition. Recently Lowe et al. (2002) solved the problem by conducting experiments with intrusive gravity currents, generated by releasing fluid of intermediate density in a two-layer stratified fluid. For small density differences, the setup is equivalent to a gravity current propagating along a free-slip boundary.

In the field, Pineda (1999) considered the transport properties of internal-bore warm fronts (buoyant gravity currents) that are occasionally observed near La Jolla, California. Different species had different distributions within the features, and measurements of currents and larval distributions supported the hypotheses that (1) larvae concentrate at the propagating fronts, and that (2) the origin of the concentrated larvae is behind the front, implying that currents in these features were faster than propagating speeds. Franks (1997) modeled the accumulation of particles ahead of a propagating front with a $2 \mathrm{D}$ kinematic model, and found that upward swimming particles originating ahead of the front accumulated behind the front. Helfrich and Pineda (2003) supported with a numerical model and lab experiments that both upward-swimming and floating particles originating behind and ahead the front can accumulate in the propagating features. However, in these models, the particles were exposed to a steady, two-dimensional gravity current. On account of the strong nonlinearity of these waves, these assumptions are very restrictive, since the flow is strongly three-dimensional (Özgökmen et al., 2004), whereas two-dimensional steady flows impose strong topological conditions which affects mixing. Indeed, the trajectories in physical space of Lagrangian particles advected by a steady and two-dimensional velocity field are in one-to-one correspondence to trajectories in phase space of particles moving under the effect of a steady Hamiltonian equal to the streamfunction of the flow (Ottino, 2004). Since the latter system is integrable, chaos (i.e. mixing) is ruled out. Moreover, the prescribed behavior of the particles was very restrictive and not very realistic. As a result, it is difficult to assess quantitatively the effective accumulation rate, nor is it possible to study the species-dependent clustering observed in the field. In this study, we follow an approach similar to Helfrich and Pineda (2003), but we take a step further and use a fully nonlinear, nonhydrostatic, unsteady and three-dimensional model to generate the flow within both gravity currents and nonlinear waves with trapped cores. Moreover, we consider recent laboratory experiments (DiBacco et al., in prep.) to model the behavior of larvae in the flow. Strictly speaking, the model is applicable to laboratory scale flows, but the results can be extrapolated to field scales.

\section{Methods}

To model the flow field associated with internal waves and gravity currents, we solve the Navier-Stokes equations in the Boussinesq approximation for a fluid of variable density

$$
\frac{D \mathbf{u}}{D t}=-\nabla p-b \mathbf{k}+\nu \nabla^{2} \mathbf{u}
$$




$$
\begin{aligned}
\frac{D b}{D t} & =\kappa \nabla^{2} b, \\
\nabla \cdot \mathbf{u} & =0,
\end{aligned}
$$

where $\mathbf{u}$ is the velocity vector, $b=g\left(\rho-\rho_{0}\right) / \rho_{0}$ the buoyancy anomaly relative to a reference density $\rho_{0}, p$ the pressure, $\mathbf{k} \equiv(0,0,1)$ the unit vector pointing in the vertical direction, $v$ the kinematic viscosity of the fluid, and $\kappa$ the diffusivity of the stratifying agent. Finally, $D / D t$ stands for the Lagrangian derivative following a parcel of fluid. The above equations are solved in a channel of depth $H$, length $L_{x}$ and width $L_{y}$. At the top and bottom boundaries free-slip conditions are applied $\partial \mathbf{u} / \partial n=\partial b / \partial n=0$, where $n$ is the direction normal to the boundary. Along the spanwise (along-front, $y$ ) direction we apply periodic boundary conditions, while in the streamwise direction we impose different conditions depending on the specific flow considered. Introducing a typical buoyancy scale $b_{\text {ref }}$, it is possible to define a velocity $U_{\text {ref }}=\sqrt{b_{\text {ref }} H} ; U_{\text {ref }}$ and $H$ can be used to make the equations nondimensional. When expressed in these units, the viscosity $\nu$ and diffusivity $\kappa$ become

$$
\begin{aligned}
\nu & =\mathrm{Gr}^{-1 / 2} U_{\text {ref }} H, \\
\kappa & =\operatorname{Pr}^{-1} \mathrm{Gr}^{-1 / 2} U_{\text {ref }} H,
\end{aligned}
$$

where Gr and Pr are the Grashof and Prandtl number respectively. Gr, the ratio of buoyancy to viscous forces, is a property of the flow, and typical field conditions are characterized by values of Gr that are $O\left(10^{14}\right)$ (Pineda, 1999), while $\mathrm{Pr} \equiv \frac{\mathrm{v}}{\mathrm{\kappa}}$ ranges from 7 to 1000 depending on whether the stratifying agent is temperature or salinity. The numerical resolution limits the largest values of $\mathrm{Gr}$ and $\mathrm{Pr}$ attainable in numerical experiments, without resorting to modeling the unresolved turbulent scales. In our study, we set Gr at $10^{8}$ and $\operatorname{Pr}$ at 10 . Analysis of buoyancy spectra showed no accumulation of energy at large wavenumbers, confirming that the resolution employed is sufficient to capture the essential dynamical scales. Table 1 lists the domain size and resolution for the runs considered here.

The setup used to maintain a steady gravity current mimics the experiments of Britter and Simpson (1978). The channel dimensions are $L_{x}=5 H, L_{y}=1.5 \mathrm{H}$. On one side $(x=0)$, a weir separates an inlet area, where fluid of density $\rho_{1}$ is injected with velocity $c_{1}$ (we will refer to this as the warm fluid), from the outflow area where an Orlanski-type boundary condition (Orlanski, 1976) allows mixed fluid to leave the domain. On the other side $(x=5 H)$, "cold" fluid of density $\rho_{0}$ is injected with velocity $c_{0}$ (Fig. 1). Physically, $c_{0}$ represents the velocity of propagation of the gravity current, while $c_{1}$ is proportional to

Table 1. Domain size and discretization used in our numerical experiments.

Type of flow

$$
L_{x} \times L_{y} \times L_{z}
$$




\section{Warm fluid inlet}

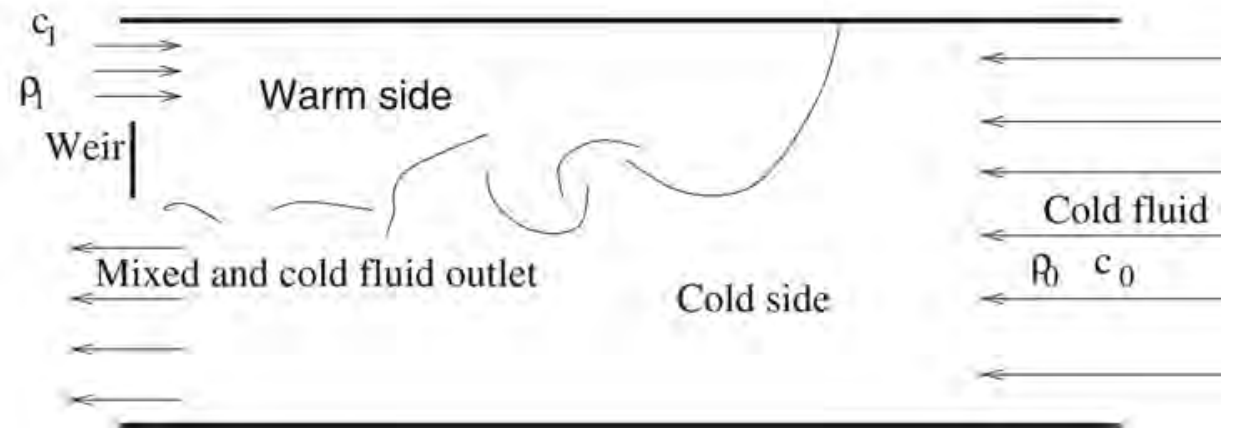

Figure 1. Domain used for the simulation of a gravity current. Warm fluid enters from the inlet with velocity $c_{1}$, mixes with the incoming cold fluid and leaves the domain below the weir. The inlet area occupies about $20 \%$ of the total domain.

the amount of mixing taking place in the turbulent flow. The gravity current was generated using a three-step approach. At $t=0$, warm fluid was injected from the inlet at constant speed while setting $c_{0}=0$. Since the injection speed was uniform in the spanwise direction, the flow remained two-dimensional, shedding Kelvin-Helmholtz vortices from the frontal region. When the advancing front reached $x=4 H$, the incoming cold flow was turned on with velocity $c_{0}$ to bring the advancing front to a halt (second step). This was also the time when we forced the flow to become three-dimensional. In a first set of experiments, this was achieved by taking the two-dimensional solution achieved at the end of the first step, and generating a three-dimensional initial condition by distorting the two-dimensional solution in the along-front direction. In a second set of experiments we instead added a small amount of turbulence $\left(u_{\mathrm{rms}}=0.5 \% c_{0}\right)$ to the incoming cold flow. This was achieved by adding white noise of given magnitude to the cold side boundary condition, and letting nonlinear interactions turn it into realistic turbulence during the time the fluid takes to travel the distance from the cold side inlet to the front. No stochastic terms were added to the Navier-Stokes equations. In both cases, $c_{1}$ was varied in real time to maintain the mean density in the channel constant. After a sufficiently long period $T, c_{1}$ was fixed at a value obtained by taking its average over $T$. Waiting a sufficiently long time also ensures that the system "forgets" the initial condition, on account of the parabolic nature of the Navier-Stokes equations. At this point (third step), the injection of particles began. This setup has the advantage over the more common lock-release experiments (e.g, Helfrich and Pineda, 2003) of possessing a statistically steady state that can be maintained for as long as necessary. Typical experiments lasted over 200 nondimensional time units.

\section{a. Particles}

DiBacco et al. (in prep.) developed an experimental setup to observe and quantify the swimming behavior of larvae, after the field observations of (Pineda, 1999) implied that 
larvae tend to swim against downwelling currents. The apparatus consists of a vertical Plexiglas cylinder through which water flows from top to bottom. Larvae are injected in the cylinder and are exposed to currents of different intensity. The observations indicate that they swim up when exposed to a downwelling current, modulating their response to maintain a constant depth up to a taxon-specific maximum swimming speed, above which the larvae are swept away. In our model, this behavior is included by setting the swimming velocity $w_{s}$ according to

$$
w_{s}= \begin{cases}0 & \text { if } w_{f}>0, \\ \min \left(w_{b}-w_{f}, 0\right) & \text { if } w_{f}<0,\end{cases}
$$

where $w_{f}$ is the vertical velocity of the fluid at the particle position, and $w_{b}$ is a taxon specific sustained maximum vertical swimming speed. Since the time scale of these type of fronts is of the order of hours at the most, we do not consider diel migration patterns. To simulate different type of larvae, we considered in our experiments five swimming velocities, from $10^{-4} U_{\text {ref }}$ to $10^{-2} U_{\text {ref. }}$. In the field, plankton tends to be localized in patches of moderate vertical thickness but extending over large horizontal distances (e.g., Mullin, 1993). For this reason, we decided to release particles at specific depths with a constant injection rate. Since the frame of reference used is stationary with the wave, it is equivalent to considering a wave sweeping plankton initially stationary ahead of it. $w_{f}$ at the particle location was calculated using a fourth-order Lagrangian interpolation scheme and a third-order accurate AdamsBashfort scheme was used to discretize in time Eq. (4). Particle injection was stopped after 100 time units, and the experiment was run for another 100 time units. Particle positions and velocities were recorded every 0.1 time units.

\section{Results}

Depending on how the front was forced to become three-dimensional (distortion of the initial condition vs. upstream turbulence), the flow settled in two distinct states, both of which are three-dimensional and unsteady, but characterized by different mechanism sustaining the production of turbulence and mixing. When the front was exposed to upstream turbulence, Kelvin-Helholtz billows were found to develop downstream of the front, breaking up as they evolved downstream and driving mixing in the wake. Without upstream turbulence, the primary instability was convective in nature and three-dimensional from the start (unlike Kelvin-Helmholtz billows, which start as two-dimensional waves). Interestingly, shutting down the turbulence upstream resulted in the disappearance of Kelvin-Helmholtz billows and the onset of the three-dimensional convective instability. Conversely, starting from a convective instability case and turning on the upstream turbulence resulted in the development of Kelvin-Helmholtz billows. It is important to notice that in both cases the resulting three-dimensional flow within the frontal region is due to the non-linear dynamics within the region. Even when turbulence is added upstream, it affects the flow near the front primarily because it promotes the development of Kelvin-Helmholtz billows, its intensity 
being too small to have any direct effect on the flow. In other words, we would expect the same flow near the front regardless of how the Kelvin-Helmholtz billows are generated, as long as they are present.

A final source of three-dimensionality within the head is represented by wave-like disturbances propagating along the frontal region in the spanwise direction. These waves interact forming cusps that are similar, at least qualitatively, to structures found along surface fronts generated by river plumes (Cooper et al., 2001) and along internal tidal bore warm fronts (Pineda, personal observation; see also Fig. 1c in Franks, 1997). A detailed analysis of the physics of the frontal region is presented elsewhere (Scotti, 2007). In the Appendix, we give a brief description of the convective instability, since it does not appear to have been described before.

Both states are likely to occur in the field, depending on the level of ambient turbulence, and have sufficiently different accumulation and transport properties to warrant a separate discussion. For simplicity, we will refer to the case with and without Kelvin-Helmholtz instabilities as $\mathrm{KH}$ and noKH.

Both $\mathrm{KH}$ and noKH propagate at similar speeds $c_{0}=0.47$, despite the fact that mixing, measured by the rate of injection of light fluid needed to maintain steady state, is $50 \%$ larger in $\mathrm{KH}$ relative to noKH. With regard to other parameters such as head maximum elevation, $\mathrm{KH}$ and noKH agree well with values reported in the literature (Britter and Simpson, 1978; Lowe et al., 2002). In our experiments the slope of the front of the head was $61^{0}$, very close to the slope predicted by the von Karman-Benjamin theory (Benjamin, 1968) and observed by Lowe et al. (2002), while in Britter and Simpson (1978) the observed value was $40^{\circ}$. This discrepancy is likely due to the fact that while in our simulations the boundary condition is free-slip along the entire upper surface, in Britter and Simpson (1978) the boundary condition in the head was no-slip (albeit with small gradient due to the head being held stationary).

Spanwise and ensemble averaged properties of the KH gravity current (Fig. 2a-d) are similar to the ones reported by Helfrich and Pineda (2003) but with some differences. The recirculation area along the back of the head is absent in the HP simulation. Also, mixing is reduced relative to the $2 \mathrm{D}$ case, as can be seen from the distribution of isopycnals. The Smagorinsky model employed in the 2D simulation likely overestimates the mixing along the density interface, resulting in a smearing of the interface and higher entrainment rates. Note however that without a closure scheme, results are generally worse (Özgökmen et al., 2004). The flow in the head is markedly three-dimensional. In fact, we routinely observed strong horizontal shear in the head.

In the noKH case, mixing is strongly reduced, and the interface between light and heavy fluid remains sharp until the convective instabilities described above set in. The 0.1 isopycnal shows the existence of an overturning region. The horizontal velocity of the fluid supplying the head is reduced. The flow in the head is almost stagnant, in agreement with the observations of Lowe et al. (2002). As the streamlines approach the head, they are deflected downward by an area of high pressure $(\mathrm{H}$ in Fig. $2 \mathrm{~g}$ ), which is actually the signature of a 

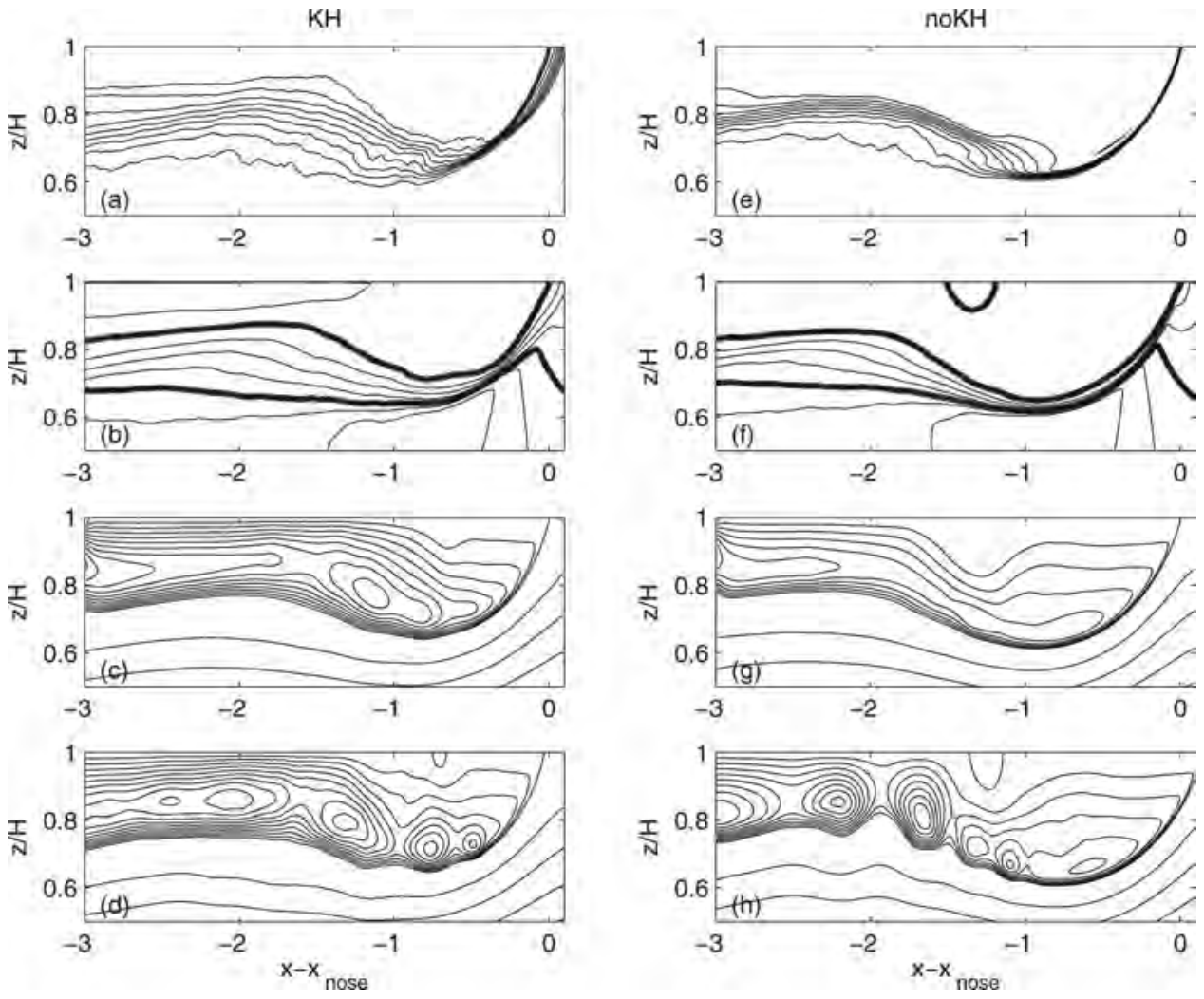

Figure 2. Ensemble-averaged structure of the head of a gravity current. Left panels are for KH currents, right panels for noKH currents: $(\mathrm{a}, \mathrm{e})$ Isopycnals; $(\mathrm{b}, \mathrm{f})$ Isolines of horizontal velocity scaled by the speed of propagation (lab frame of reference); $(c, g)$ Averaged streamlines in a frame of reference moving with the head; $(\mathrm{d}, \mathrm{h})$ Streamlines in a frame of reference moving with the head at a specific instant in time (averaged in the along-front direction).

persistent recirculation area (Fig. 2h). While not as strong as in the KH case, the flow in the head remains three-dimensional, especially when spanwise-propagating waves are present. Thus, $\mathrm{KH}$ and noKH offer different environments. We will see that this leads to different entrainment and detrainment.

\section{a. Entrainment rate and characteristic residence time}

The number of particles contained in the head $N_{p}(t)$ reflects the balance between entrainment $E$ and detrainment $D$ rates, that is

$$
\frac{d N_{p}}{d t}=E-D .
$$


We model $E=\alpha F_{p}$, where $F_{p}$ is the incoming flux of particles and $0 \leq \alpha\left(w_{b}, z_{0}\right) \leq 1$ is the entrainment rate, which we assume to depend on the swimming speed $w_{b}$ and upstream depth of the particles $z_{0}$. In our experiments $F_{p}$ is fixed, while in the ocean is given by $\rho_{p}$ (the concentration of particles) times either $c_{0}$, for particles ahead of the front, or $c_{1}$, for particles behind it.

For the detrainment rate we assume a linear relationship $D=\tau\left(w_{b}, z_{0}\right)^{-1} N_{p}$, with $\tau$ a characteristic residence time. In a realistic setting, we expect $N_{p}$ to grow, possibly reach a steady state and eventually decay, since plankton in the field is patchy, and thus $E \neq 0$ only for a limited period of time. Efficient transport is possible only if both $\alpha$ and $\tau$ are large. As the front approaches a patch of plankton, we expect the number of particles in the head to increase linearly with time at the beginning $(t / \tau \ll 1)$, after which the rate of growth slows down. Depending on how long the source is active, which in the field is proportional to the concentration of plankton, and the number and spatial extension of the patches, $N_{p}$ may or may not reach the saturation limit $\alpha F_{p} \tau$, before dropping when the incoming flux stops. Figures 3 and 4 show the observed evolution of $N_{p}$, defined as the number of particles in the region containing the head $\left(-2<x-x_{\text {nose }}<.2\right.$ and $\left.-.45<z<0\right)$, for the noKH and KH cases. For particles released ahead, the abrupt drop immediately after the end of the injection period $(t=100)$ can be easily explained. In the absence of any entrainment mechanism, particles ahead still move through the measuring window, which they traverse in a time roughly equal to $L_{x} / c_{0}$. Within this time interval, the number of particles inside the window will increase at the maximum rate $F_{p}$. Similarly, after the injection ends, it will take an equal amount of time for the particles that are not entrained to leave the domain. If the number of particles that are not entrained is large, $N_{p}$ will drop linearly during this transient time.

In general, with appropriate choice of characteristic time and entrainment rate, the model fits quite well the $N_{p}$ profile obtained from the numerical simulation (Fig. 5). The characteristic time was calculated using the data from the decay portion of the profile (after $t=120$ ). The entrainment rate was calculate fitting the data between $t=20$ and $t=100$ in the KH case, and $t=40$ and $t=100$ in the noKH case.

In the $\mathrm{KH}$ case, the characteristic residence time depended only on the swimming speed of the particle (Fig. 6). Since the flow within the head is very energetic and homogeneous, particles are exposed to similar conditions regardless of their position within the head. In this case, the characteristic time will depend solely on the height of the head $H$, the rms value of turbulent fluctuations $u^{\prime}$ and the swimming speed $w_{b}$. Dimensional analysis then dictates that

$$
\frac{\tau u^{\prime}}{H}=f\left(\frac{w_{b}}{u^{\prime}}\right) .
$$

A simple exponential profile $f(x)=11 e^{10 x}$ fits the data quite well. Unlike the characteristic time, the entrainment rate depends also on depth and position relative to the front (Fig. 7). Not surprisingly, faster swimmers have higher rates, and so do particles behind the front. While $\alpha$ changes considerably with swimming speed, the ratio $\alpha\left(z=z_{1}\right) / \alpha(z=0)$ is 

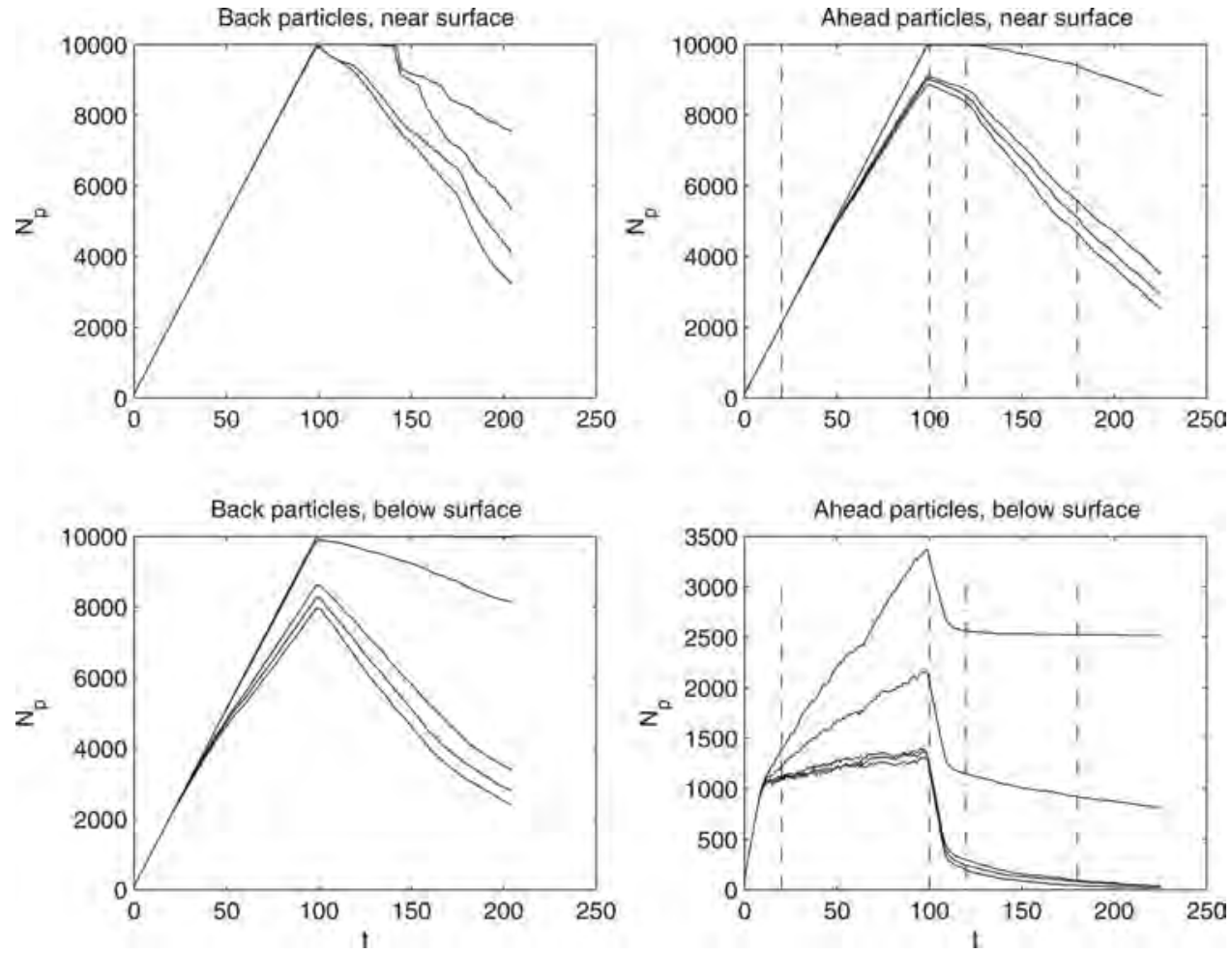

Figure 3. Number of particles in the frontal region as a function of time for particles released ahead (left panels) and behind a noKH gravity current (right panels). Different curves are for different swimming speeds, ranging from $10^{-4}$ to $10^{-2} U_{\text {ref }}$. The vertical dashed lines bound the section of the time series used to determine the entrainment rate (only shown for particles released ahead), while the dash-dotted lines mark the interval used to estimate the characteristic time.

only weakly dependent on the swimming speed over the range considered for particles initially ahead of the front. This suggest the following conceptual scenario: A particle in the cold side, which happens to be in the proximity of the cold/warm interface has a certain probability $P$ of crossing the interface in a given interval of time, which depends on the swimming speed and the level of turbulent fluctuations near the interface. The time $T$ a particle is near the interface depends on the original depth $z_{0}$, being maximum near the surface and decreasing to zero when $z_{0}>H$. In fact, by assuming a simple model for the mean streamwise velocity along the head (Benjamin, 1968), it is possible to show that $T \sim \sqrt{H}-\sqrt{z_{0}}$. The entrainment rate is then proportional to $P T$.

Without Kelvin-Helmholtz instabilities, both $\tau$ and $\alpha$ are higher. In fact, with the exception of subsurface particles released upstream of the front, which have entrainment rates similar to the $\mathrm{KH}$ case, the entraiment rate is always $100 \%$. For the characteristic residence time there does not appear to be a universal profile. An exponential profile fits the 

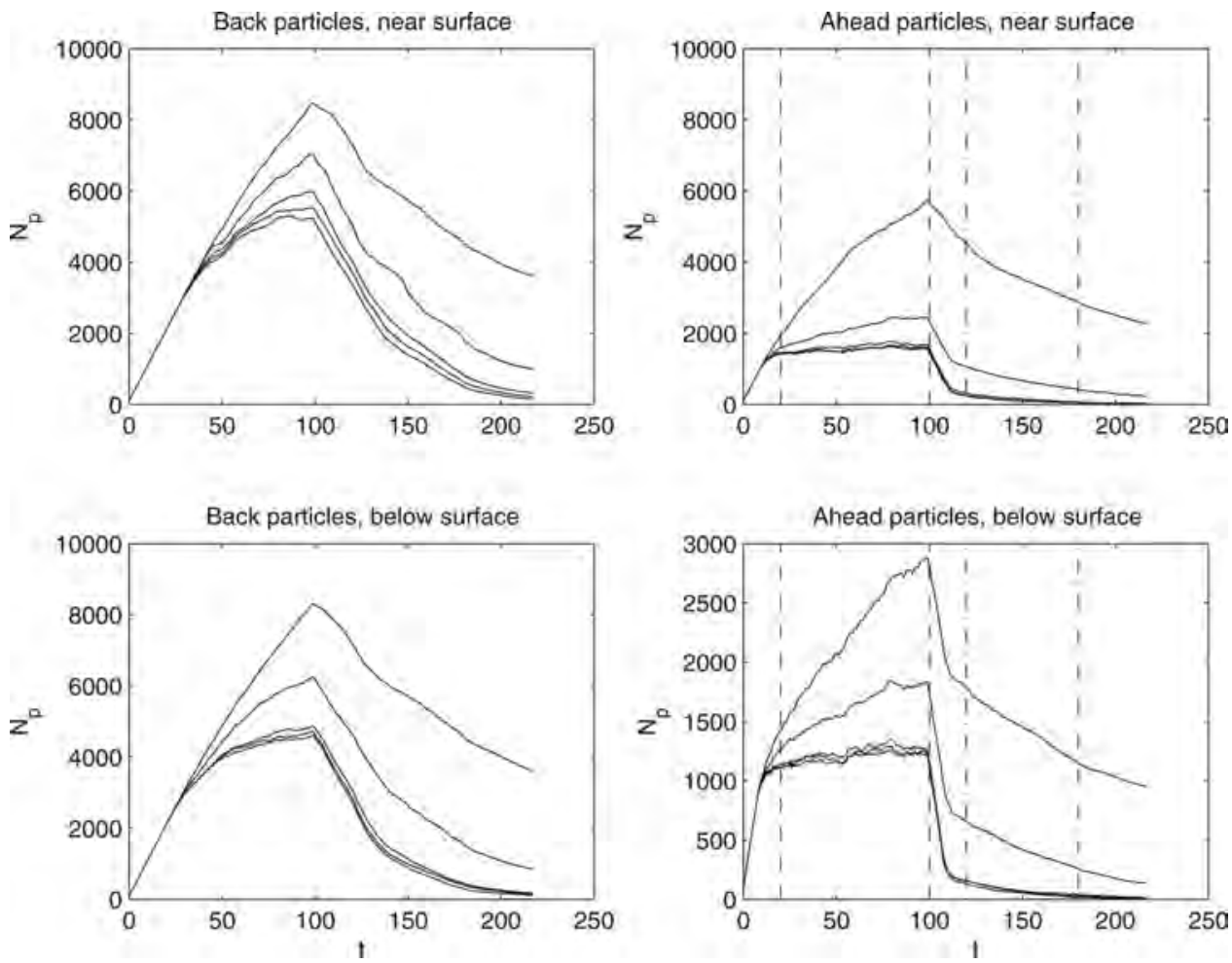

Figure 4. Number of particles in the head region as a function of time for particles released ahead (left panels) and behind a KH gravity current (right panels). See Figure 3 for details.

characteristic time of particles with different swimming speed released at the same point, but the coefficients depend on the position relative to the wave (Fig. 8).

\section{b. Spatial pattern of particle accumulation}

$i$. noKH case. The concentration of particles within the head was far from uniform. Particles initially near the surface in the warm side of the gravity current accumulated near the small near-surface recirculation area on the warm side of the head (Fig. 9, $x, y$ about -1.4, -0.1). Virtually no particles were found near the front. Particles initially in the subsurface area of the warm side were instead found preferentially in the recirculating area at the base of the head $(-0.4,-0.3)$, with higher concentrations found towards the leading edge. The exception was the fastest swimmers $\left(w_{b}=0.01\right)$, which were found on the upstream side of the head $(-1.4,-0.1)$ and, to a lesser extent, near the leading edge of the head $(0,-0.1)$. Near surface particles coming from the cold side were found in the same recirculating area occupied by subsurface warm-side particles $(-0.4,0.3)$, with the exception of the strongest swimmers (Fig. 10). The strongest swimmers accumulated at the leading edge 

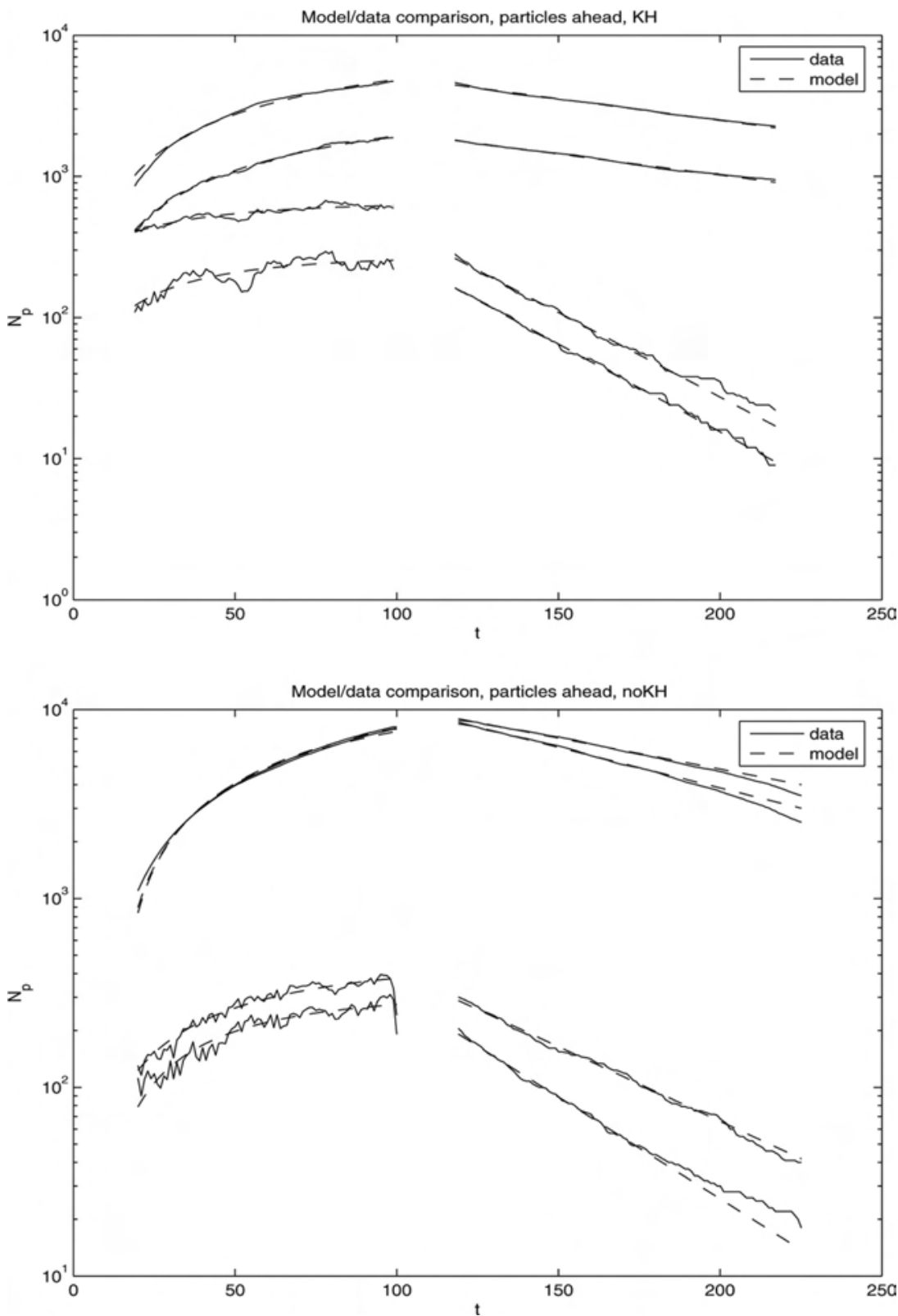

Figure 5. Comparison between modeled (Eq. 5) and computed particle concentration near the front. (a) KH case and (b) noKH case. For clarity, only four combination of swimming speeds and release depths are shown. In the inset "data" refer to the results of the numerical simulation, "model" to the fit with Eq. 5. For particles released behing the front a similar good fit is obtained. 


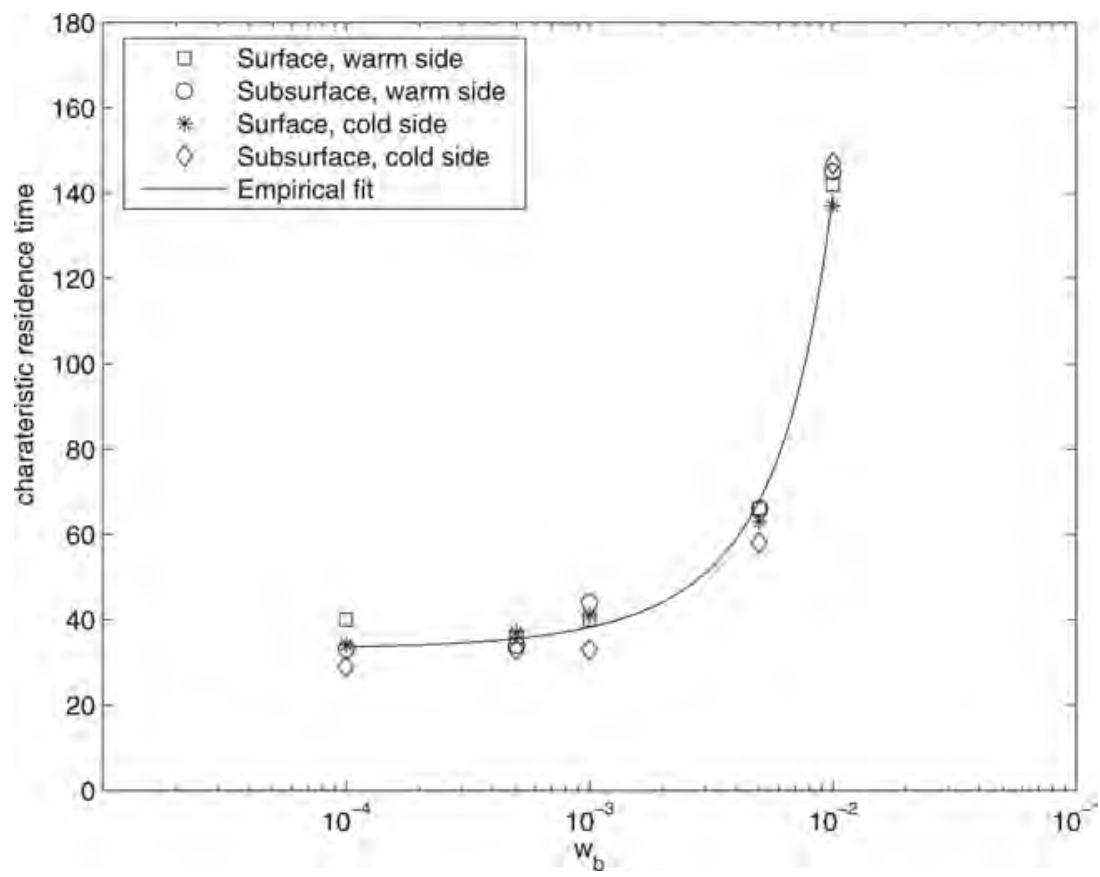

Figure 6. Characteristic residence times for particles released in a $\mathrm{KH}$ gravity current.

of the gravity current. Subsurface cold-side larvae concentrated along the separatrix which divides the cold from the warm side, with higher concentrations near the leading $(0,-0.2)$ and trailing $(-1.4,-0.4)$ edge of the head (Fig. 10).

While it is reasonable to expect a sorting of the larvae in the streamwise-vertical plane, it was surprising to discover large variation in concentration along the spanwise direction. It appears that the three-dimensional nature of the flow within the head causes particles to accumulate in hot spots along the spanwise direction. This phenomenon was independent of the swimming velocity of the particles, though it was more pronounced for larvae found near the surface. In fact, for all cases considered, Probability Density Functions of particle concentration were highly skewed, with broad tails, implying that a large fraction of entrained particles were concentrated in a few hot-spots. Figure 11 summarizes the observed pathways of entrainment in a noKH gravity current.

ii. KH case. The behavior-dependent pattern of segregation observed in the noKH case is found in the KH case as well, though with different accumulation loci. For surface particles, both in the warm and cold side, the highest accumulation is found near the leading edge (Figs. 12 and 13), except for the fastest swimmers coming from the cold side, which are spread along the frontal region of the head. Weak and moderate subsurface swimmers from the warm side are more uniformly distributed within the head, while fast swimmers 


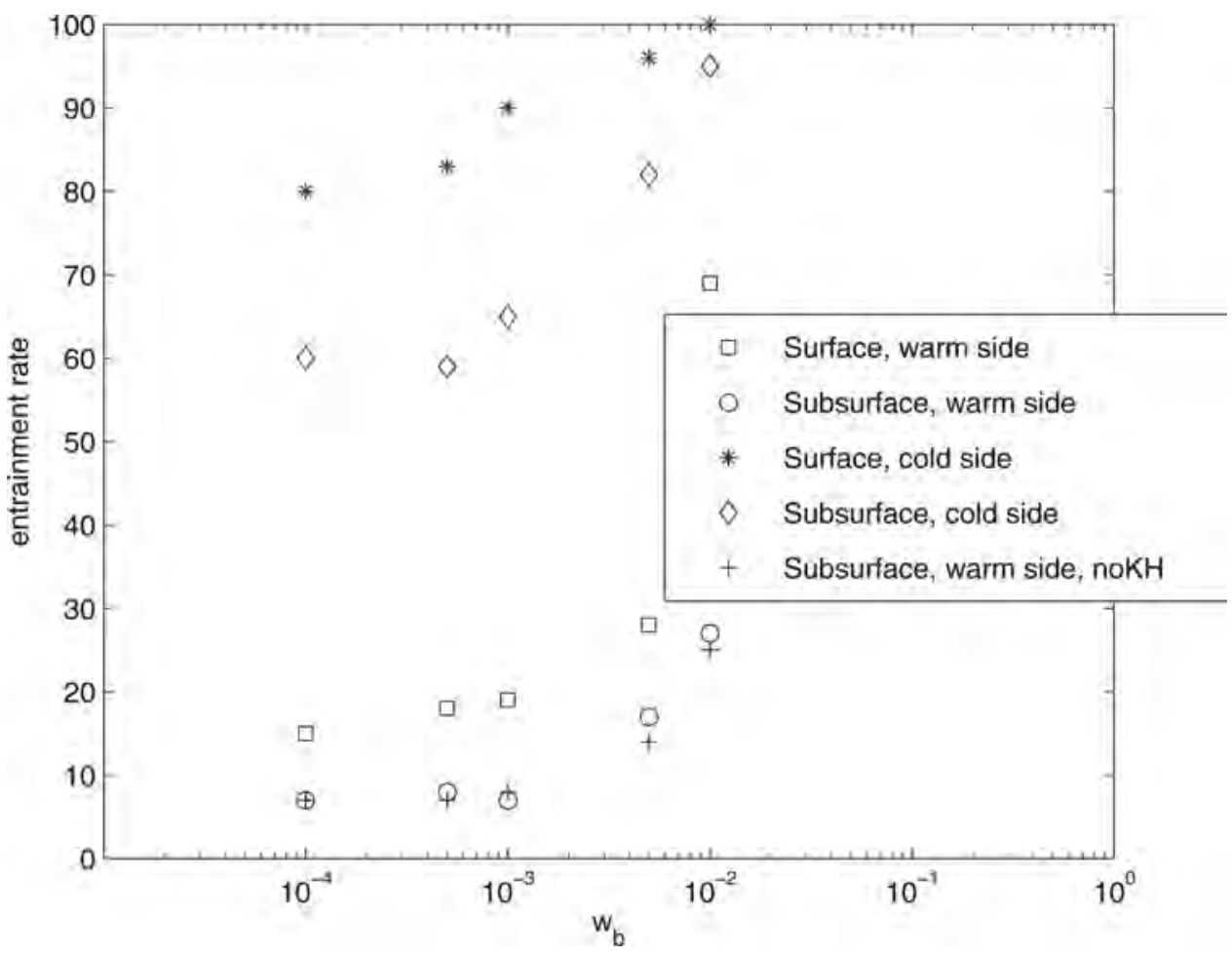

Figure 7. Entrainment rates as a function of swimming speed for $\mathrm{KH}$ currents, except when otherwise indicated.

concentrate at the surface frontal region. Conversely, fast cold side subsurface swimmers tend to occupy the entire head region, while slower particles concentrate along the front and along the turbulent backside of the head. Along the spanwise direction particles were distributed more uniformly than in the noKH case, but still with large variations in concentration. Figure 14 shows a snapshot of strong swimming particles that were initially released near the surface behind the front. Particles accumulate along filaments where downwelling occurs. The pattern shows clearly how downwelling occurs both near the front, where it is expected, but also along the rear section of the head, where warm fluid is pushed downward driven by mixing. Figure 15 summarizes the spanwise averaged expected path of entrainment for the KH case.

\section{c. Eulerian concentration and particle persistence in fronts}

In the preceeding sections we have established the validity of Eq. 5 in describing how particles accumulate in the front. Physically it implies that a front is akin to a more or less "faulty" vacuum cleaner. Unlike an efficient vacuum cleaner, the front may not pick up all of the particles along its path, and even those that are collected eventually "leak out." It is 


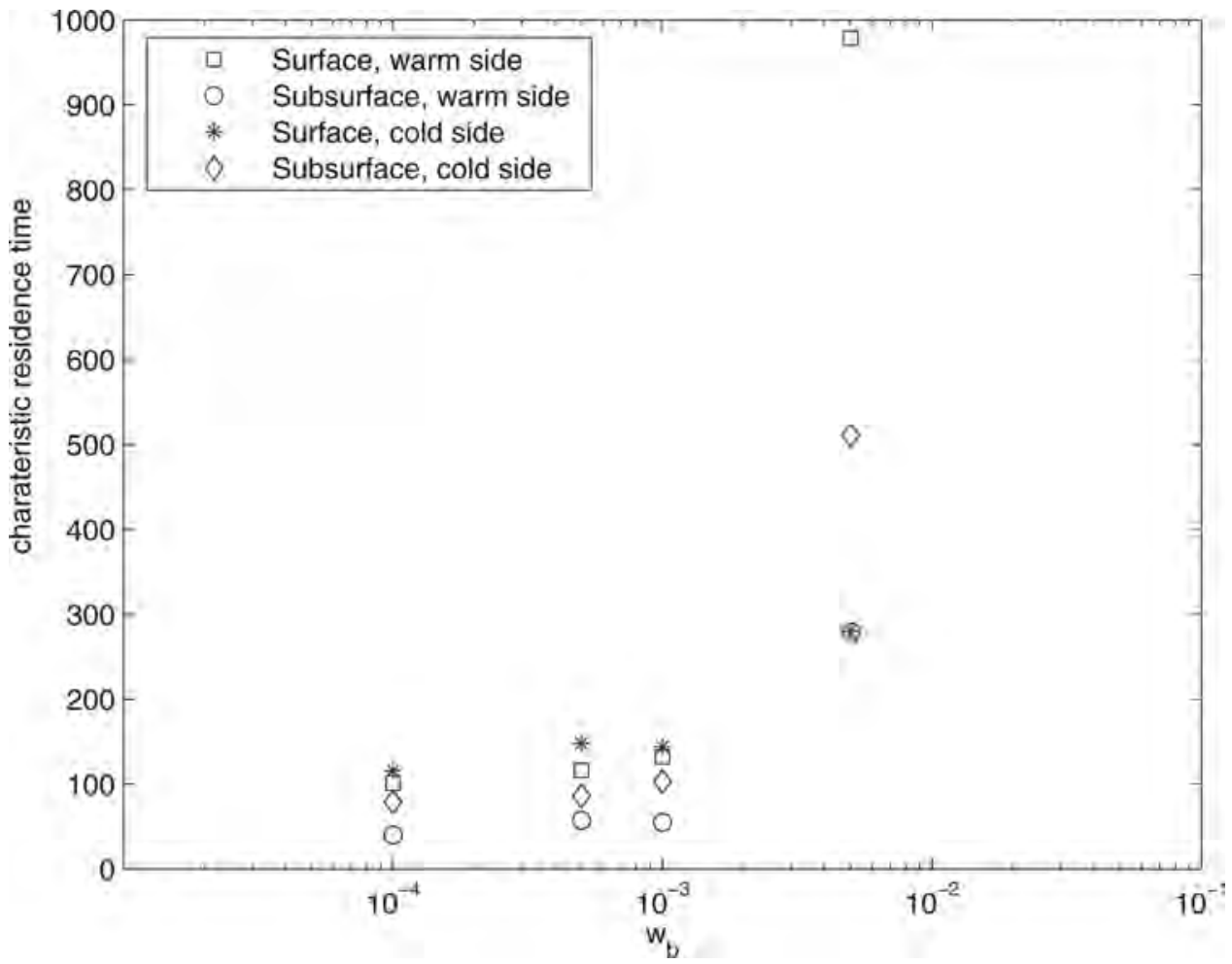

Figure 8. Characteristic residence times for particles released in a noKH gravity current.

therefore appropriate to ask how a patch of plankton evolves under the action of such a front. For simplicity, we consider only the case of plankton initially ahead of the front. To carry such analysis it is convenient to consider the two limiting cases of a patch whose extension $L$ along the direction of propagation is small or large relative to $\tau c_{0}$ (the reason why this is the appropriate reference scale will become clear immediately). Also, we revert to lab units. First consider a narrow patch, characterized by a certain density of plankton $C_{\infty}$. When the front sweeps the patch, the number of particles accumulated in the front climbs rapidly to $N_{0}=N(0)=\alpha C_{\infty} L$, after which particles begin to leak out. In a short time $d t, N(t) d t / \tau$ particles leave the frontal area, while the front advances $d x=d t / c_{0}$, hence the density of particles behind is $C(x)=N\left(\left(x-x_{0}\right) / c_{0}\right) / \tau c_{0}$, where $x_{0}$ is the original position of the patch and we have expressed elapsed time as a function of distance travelled. Since $N(t)=N_{0} \exp (-t / \tau)$, the concentration at position $x$ behind the front is given by $C(x)=\alpha C_{\infty} L \exp \left(-\left(x-x_{0}\right) / \tau c_{0}\right) / \tau c_{0}$, whereas the concentration of particles within the head is $C_{h}=\alpha C_{\infty} L \exp \left(-\left(x-x_{0}\right) / \tau c_{0}\right) / h, h$ being the size of the frontal region where particles accumulate (typically, of the order of twice the total depth, see Fig. 2). Figure 16 shows profiles at three times after the front has swept the patch. After a time long relative to 

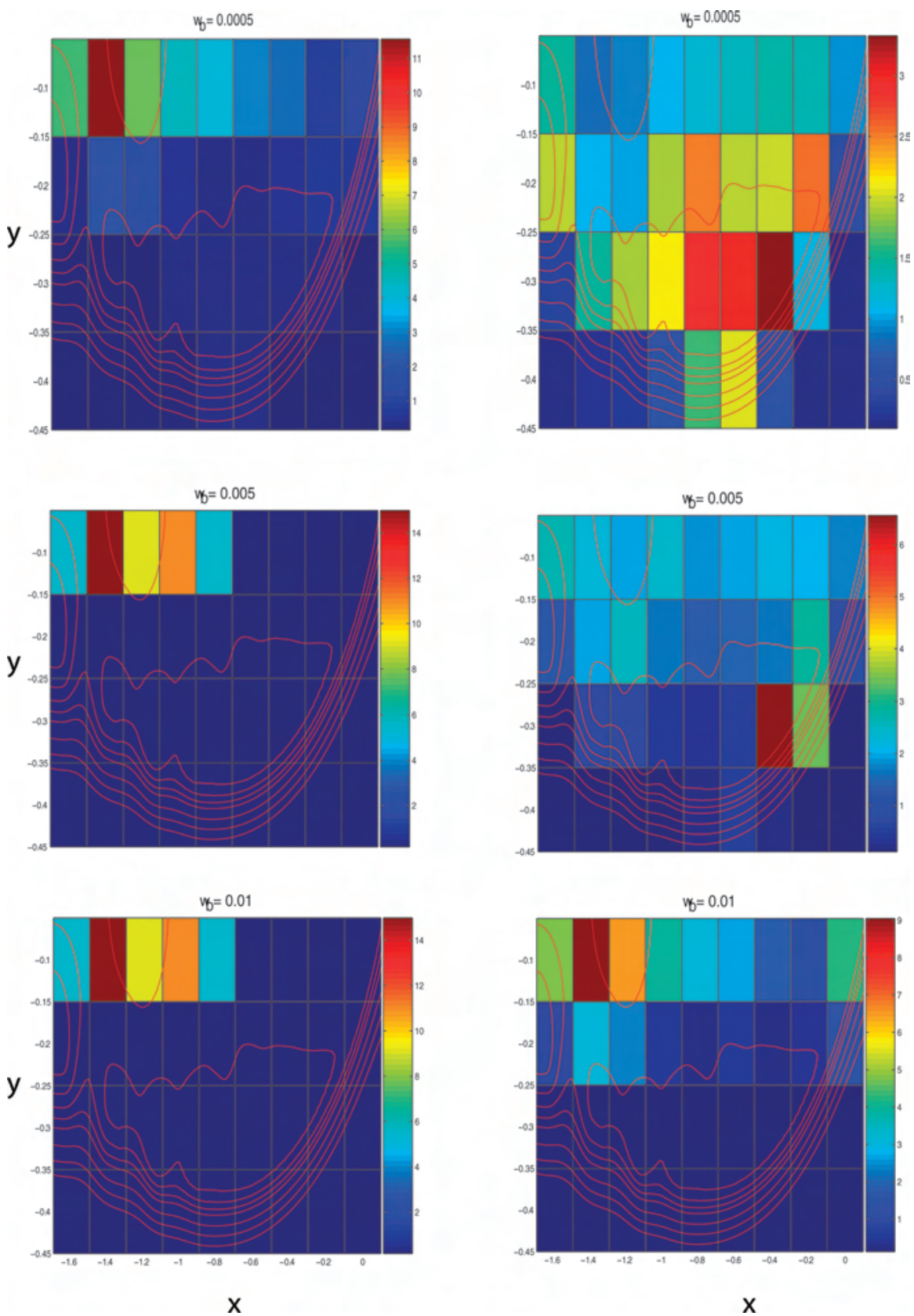

Figure 9. Concentration of particles released behind the front, noKH experiments. The left column shows concentration of particles released near the surface, the right column is for particles released subsurface. The concentrations are averaged in the spanwise direction and normalized with the mean concentration (the total number of particles divided by the volume of the head). 

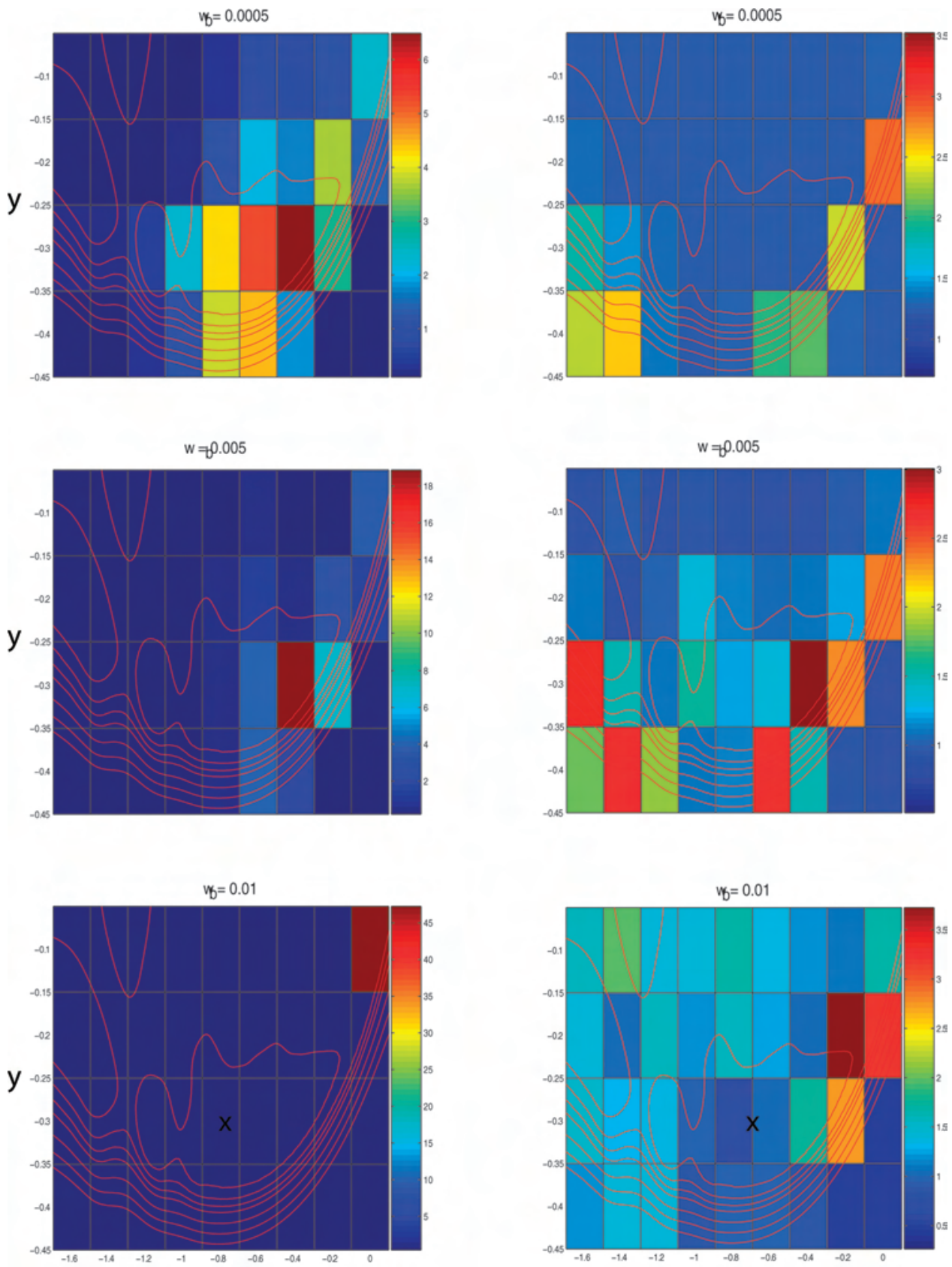

Figure 10. Same as figure 9 but for particles initially ahead of the front. 


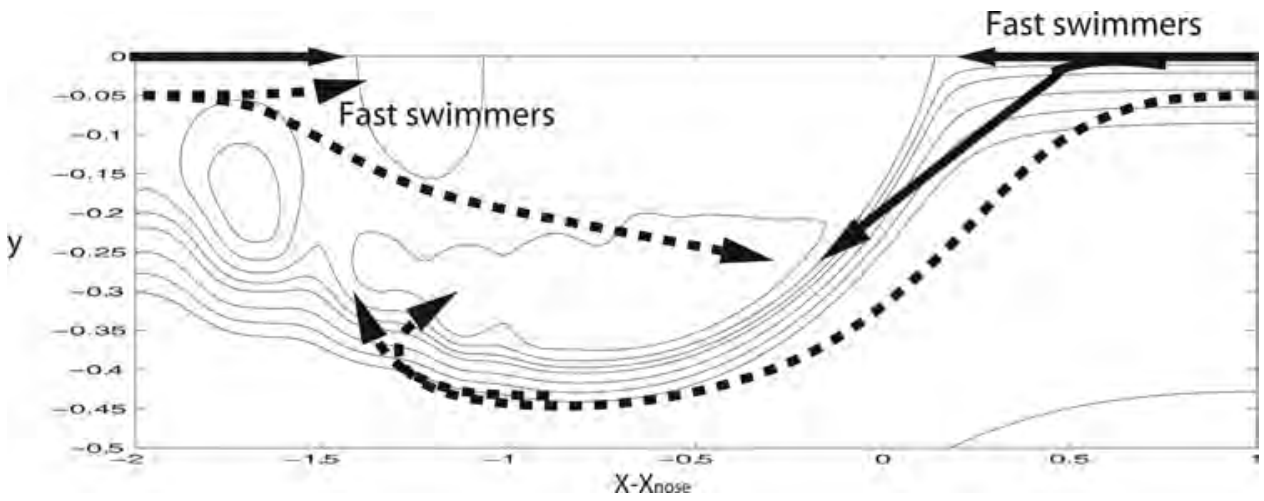

Figure 11. Pathways of entrainment in a noKH front. Solid lines are for near-surface particles, broken lines for subsurface particles.

$\tau$, all of the particles have been left behind. The centroid of the resulting distribution can be easily calculated as $x_{t}=\int_{x_{0}}^{\infty}(x-x 0) C(x) d x / \int_{x_{0}}^{\infty} C(x) d x=\tau c_{0}$ and can be interpreted as the average distance travelled by the particles. If, on the contrary, the patch extends over a long strech, the concentration of particles within the head has time to reach its steady state value given by $\alpha C_{\infty} c_{0} \tau / h$. Since $c_{0} \simeq U_{\text {ref }} / 2$ and $h \simeq 2 H$, the relative increase in concentration can be readily estimated from the data presented above, once the swimming speed is knwon. For example, strong swimmers initially near the surface on the cold side will reach a concentration inside the front 80 times larger than the background. Behind the front, the concentration is equal to the concentration ahead of the front, since the number of particles in the frontal region is constant, and thus as many particles are ejected as they are entrained. However, there is still net transport of particles forward, again given by $\tau c_{0}$, since particles spend on average a time $\tau$ within the frontal region. When particles are approaching from behind, the picture remains the same, with the only difference that the reference length for the patch is $\tau c_{1}$. To summarize, from an Eulerian point of view, the front acts as a local concentrator, with the largest concentration extending a distance of the order of twice the total depth from the leading edge, in agreement with aerial photographs of swimming dinoflagellates in fronts (see, e.g. Fig. 2(b) in Franks, 1997).

\section{d. Swimming statistics}

While entrainment in a nonlinear internal wave allows larvae to move across large horizontal distances, it requires sustained effort. In order to quantify the energy expenditure, we assumed a linear relationship between drag $F_{d}$ and relative velocity $w_{r}$ (Stokes law) and integrated $F_{d} w_{r}$ over the duration of the experiments. The result was normalized by the energy required for constant swimming at speed $w_{b}$ over the same length of time, to obtain the fractional energy expenditure (FEE). FEE is thus a measure of how energy-friendly the tranport process is. A FEE close to zero means that the larvae moves with the front almost 

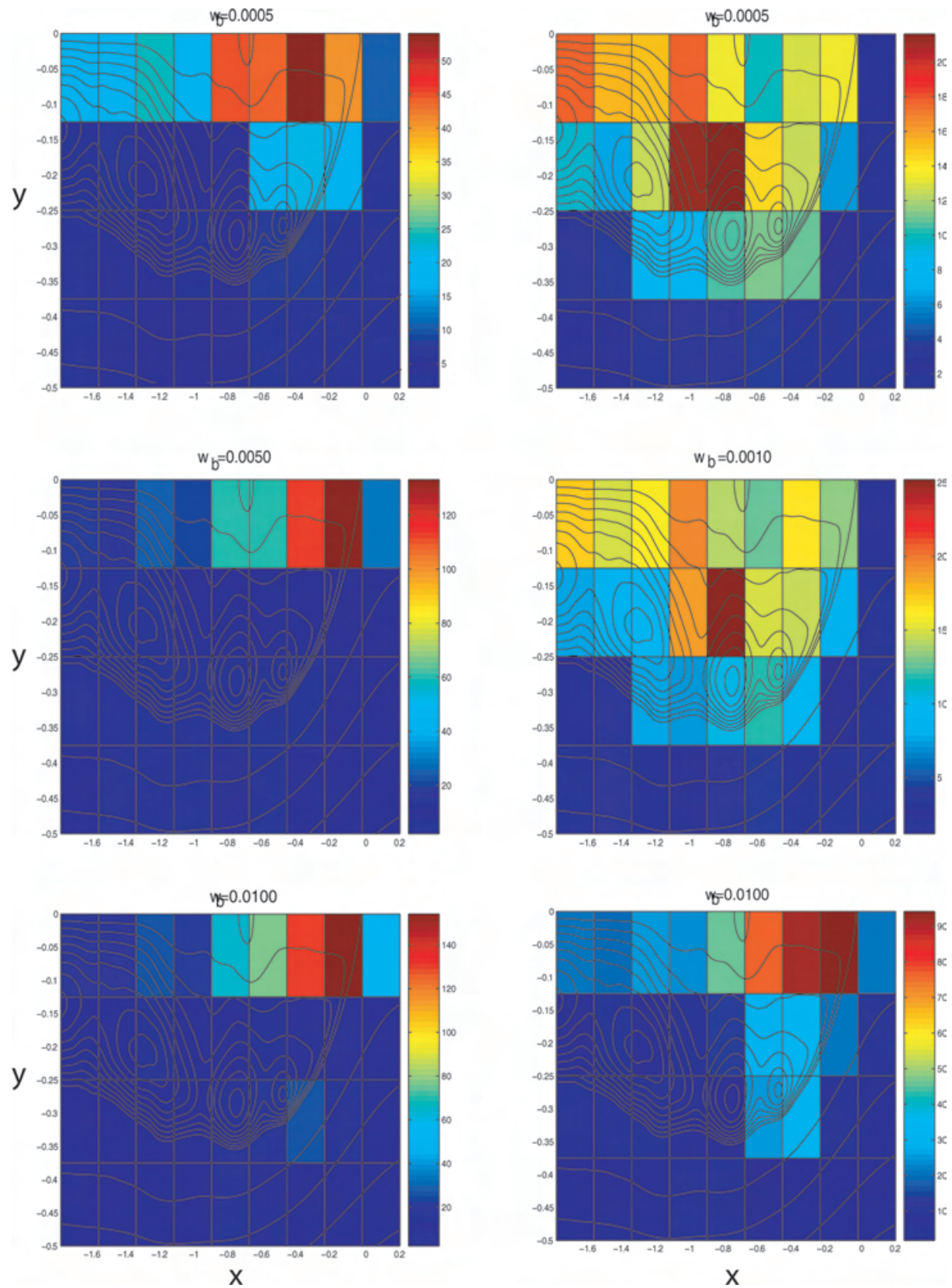

Figure 12. Concentration of particles released behind the front during KH experiments. The left column shows concentration of particles released near the surface, the right column is for subsurface particles. The concentrations are averaged in the spanwise direction and normalized with the mean concentration (the total number of particles divided by the volume of the head). 

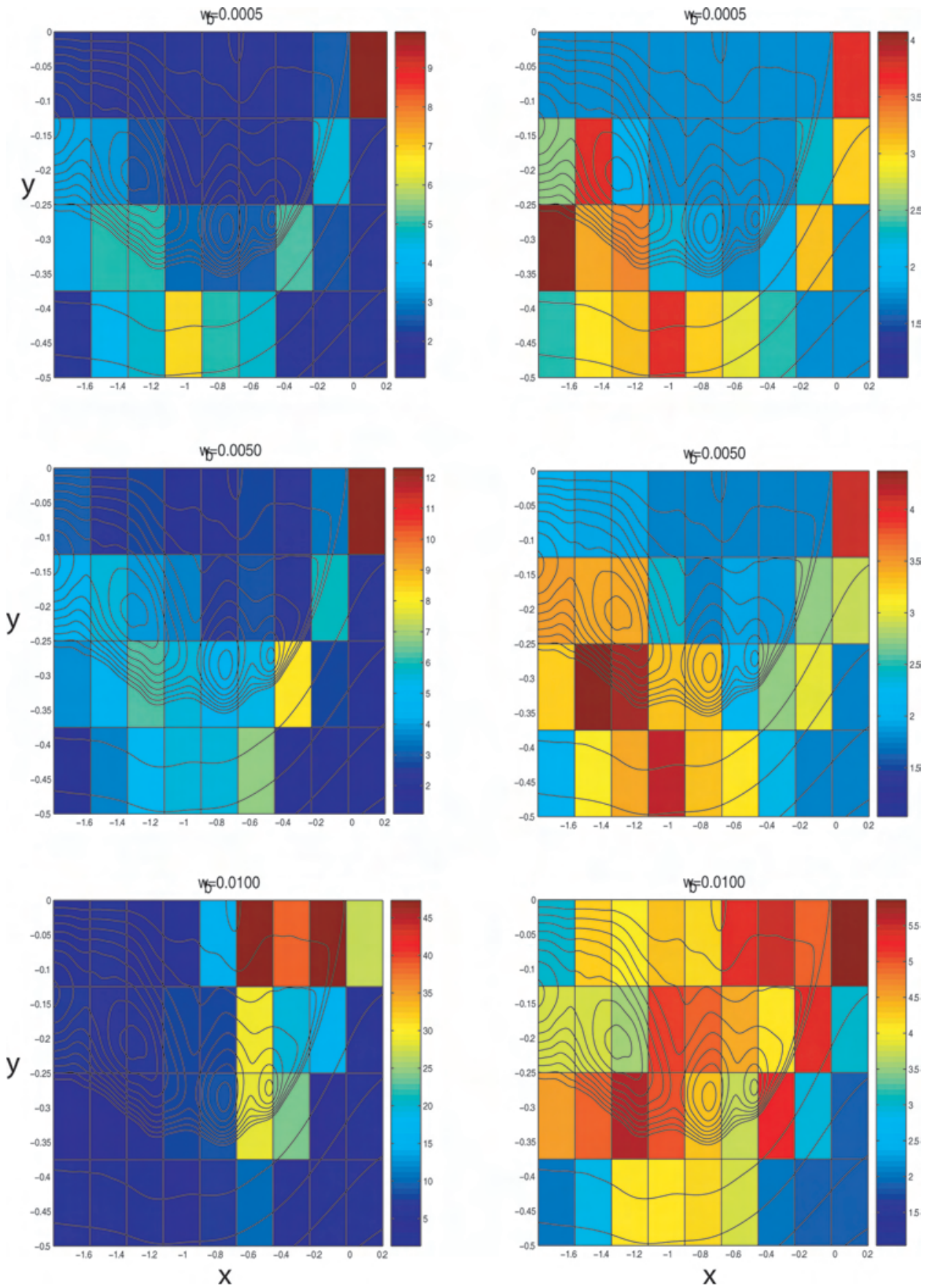

Figure 13. Same as figure 12 but for particles initially in the cold side. 


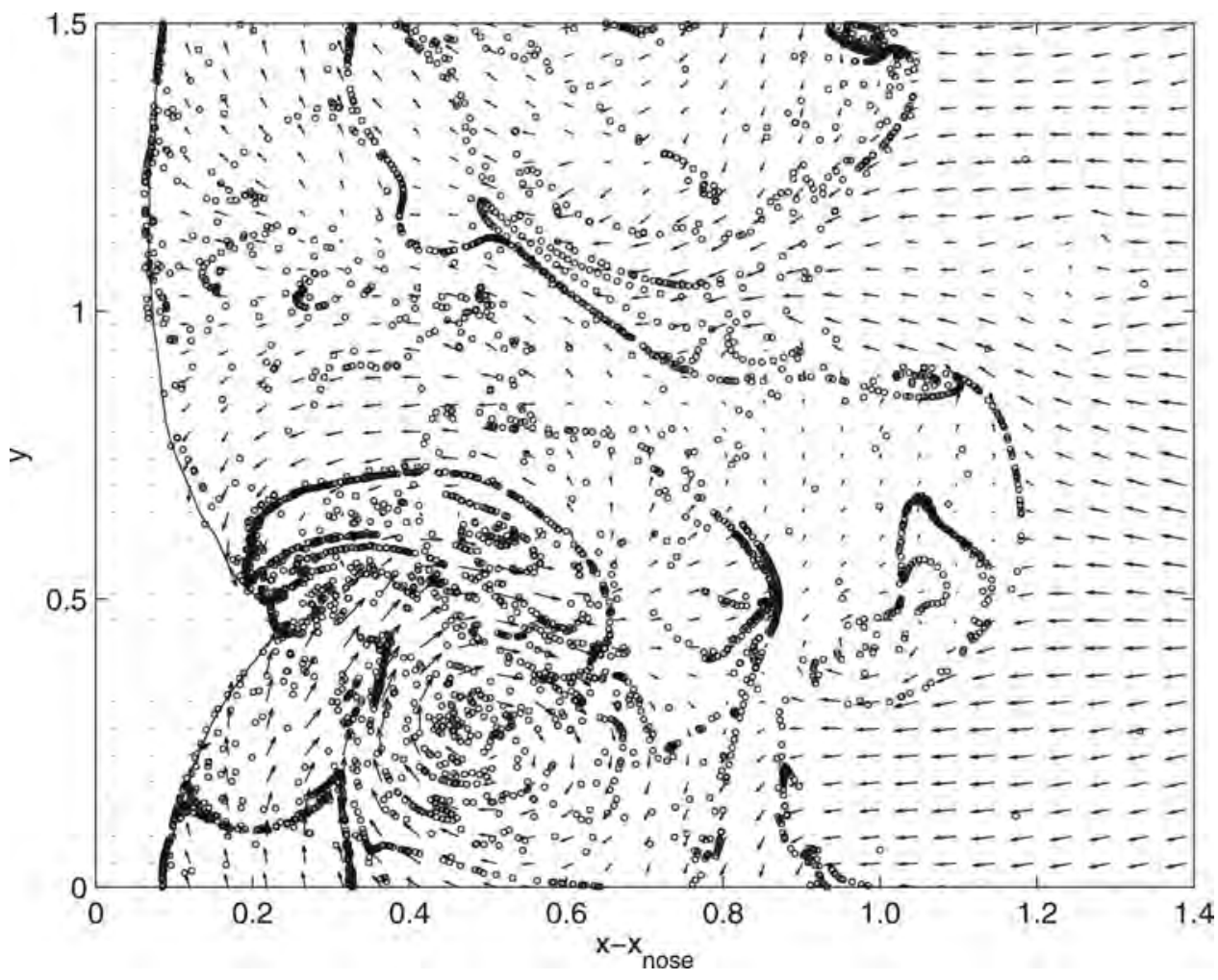

Figure 14. View from above of strong swimming particles accumulated in the frontal region, KH case. The particles were initially released near the surface behind the front. Also shown is the velocity field at the surface (arrows) in a frame moving with the front, and the location of the front (solid line to the left).

\section{Fast swimmers}

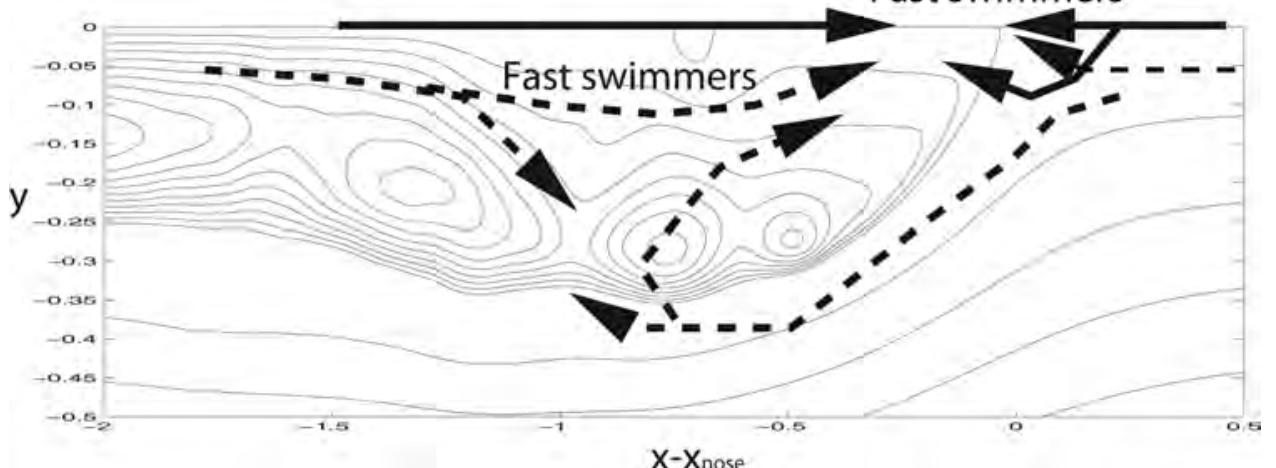

Figure 15. Entrainment pathways when $\mathrm{KH}$ instabilities are present. Solid lines are for near-surface particles, broken lines for subsurface particles. 

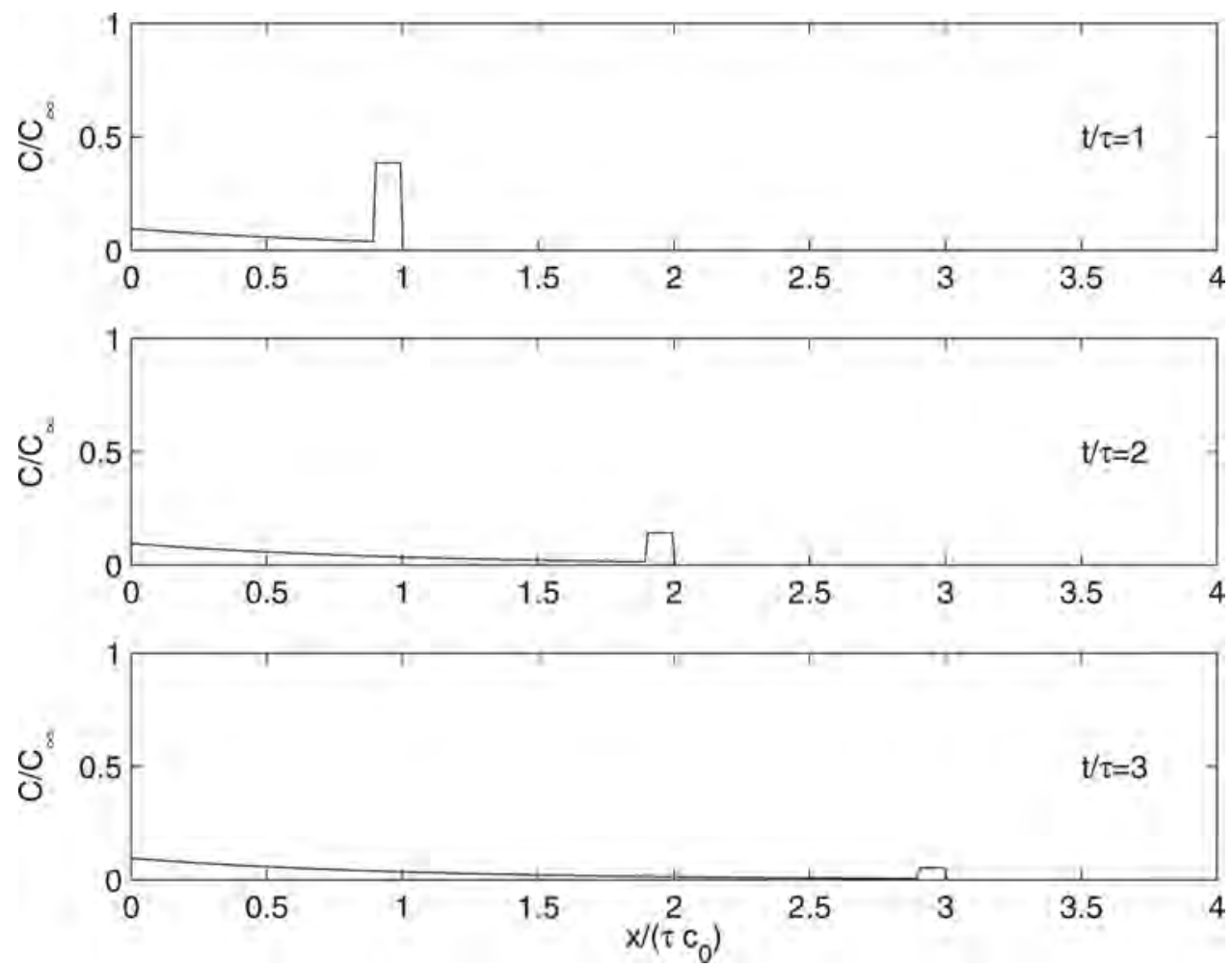

Figure 16. Concentration profiles of a small patch which at time $t=0$ is just ahead of a front. The concentration is normalized with the concentration in the patch prior to the arrival of the front. The extension of the patch was assumed to be equal to the width of the frontal region, and $L / \tau c_{0}=0.1$.

effortlessly, while a FEE of $100 \%$ means that the larva needs to swim constantly to keep up with the front. Statistics were accumulated over the particles that at the end of experiment were still in the head region. In all cases, FEE was $60 \%$ or less. In fact, the faster the swimming speed $w_{b}$, the lower the fraction of energy spent. For subsurface particles, FEE was roughly independent of the origin (ahead or behind) and type of experiment (noKH vs. $\mathrm{KH})$. The overall difference in FEE was also small. For particles near the surface the swimming effort varied considerably with position and type of experiments. Particles released ahead of of the front experienced a similar FEE up to $w_{b}=0.005$, being about $55 \%$ in the noKH case and $45 \%$ in the KH case. The fastest swimmers in the noKH case were able to nudge themselves at the front, very close to the surface (where vertical velocities are small because of the free-slip boundary condition), and thus were able to be transported essentially effortless. On the other hand, turbulent fluctuations present in the $\mathrm{KH}$ case forced even the fast swimmers to spend energy to remain in the frontal region (see Figs. 10 and 13) though proportionally less that the weakest swimmers. Particles approaching the front from the cold 


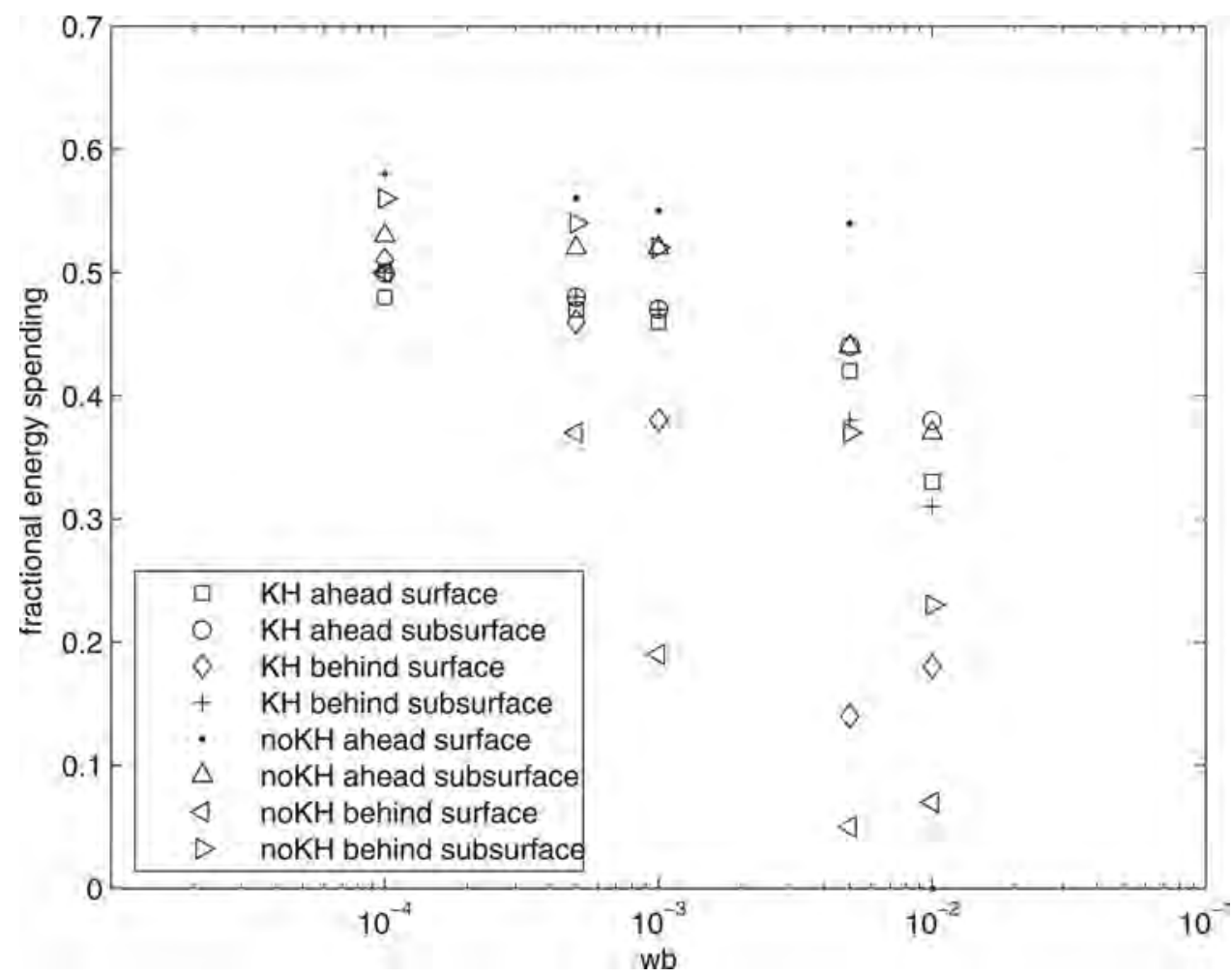

Figure 17. Fractional energy expenditure as a function of swimming velocity, experiment type and initial position.

side experienced higher FEE in KH experiments than in noKH, reflecting the higher level of turbulent fluctuations in the warm side when KH rolls are allowed to develop (Fig. 17).

If the particles were to travel the same distance unassisted by the front, the energy expenditure would be $O\left(U_{f} / w_{b}\right)$ larger. Thus, it is energetically convenient to be able to hitch-hike a moving front.

\section{Discussion and conclusions}

In this paper, we have considered the accumulation of larvae in fronts exposed to the fully nonlinear, non-hydrostatic and three-dimensional flow of a propagating front. The two main ingredients are a model for the fluid flow based on the three-dimensional Navier-Stokes equations, and a model for the behavior of larvae. A stringent test of the model would be to compare the results with field data, which are not available. Thus, to asses the validity of the approach vis-a-vis the real world, we criticize the different elements. The properties of our solution compare well with available lab-scale experiments. However, we need to determine whether we can extrapolate our results to oceanic values of $\mathrm{Gr}$ (and $\mathrm{Pr}$, if stratification 
is dominated by salinity). To this question we think we can answer a conditional yes. The experience accumulated in the computational fluid dynamics community over the last two decades shows that once a sufficiently high value of the Reynolds number (whose role here is played by $\mathrm{Gr}$ ) is reached, then we can expect statistical equivalence of solutions at different values of the Reynolds (or Grashof) number provided the solutions are properly normalized (e.g, see the discussion in Moin and Manesh, 1998). At the Grashof number considered, the flow is turbulent, and is characterized by mixing properties (not discussed here) that are typical of well developed turbulence. Hence, we are confident that the flow is realistically represented in our model. The second main element of our study is the model for the plankton behavior. Clearly, in the field planktonic larvae may respond to a gamut of stimuli, which might elicit compensating responses and be adaptive in various circumstances. For example, Franks (2001) suggested that increased food ingestion by larval fish with enhanced turbulence could be explained by turbulence avoidance behavior by larval fish and their prey, and not necessarily by turbulence increasing the encounter rate between predators and prey. Of the possible responses, we have selected a particular one (swimming against a downwelling current), which is observed in the field and might be adaptive because swimming against the currents modulates the onshore transport of larvae to adult habitats. Moreover, this behavior has been quantified and studied in isolation in the lab (DiBacco et al., in prep.). The goal of this study is not to show that plankton possess an innate drive to join the first passing gravity current, but that a simple behavioral response is enough to allow plankton to use fronts for long-distance travel. We have found that there are important qualitative and quantitative differences between strictly two dimensional fronts and fully three dimensional cases. In the latter case, different primary instabilities trigger the transition to a turbulent state behind the head, and cause a different environment in which plankton founds itself. The strong spatial structure in the along-front direction (Figs. 4 and 6), with the associated variability in accumulation, has important implication for sampling in field conditions. For example, it underscores the risks of drawing conclusions on the accumulation characteristics of these phenomena, as well as on the larval behaviors required to accumulate in these, from sampling single events, as conclusions would be different depending on which along-front section is sampled. In cases where the alongshore structure cannot be resolved, the best approach might be to sample several events (e.g. several independent fronts) and then average the results, though this averaging approach would tend to brush out the fine spatial differences.

Despite the complexity of the flow, bulk transport properties can be described with a relatively simple model, characterized by two parameters with a simple physical interpretation. One is related to the probability that a particle gets entrained in the head of the current, and the other measures the residence time of particles in the head. Transport efficiency depends on both of them being large. It appears that plankton capable of sustained swimming speeds on the order of a small fraction of the speed of propagation of the front can achieve this goal. Furthermore, our analysis shows that planktonic larvae do not have to swim constantly to remain at the head, and that faster swimmers require proportionally less energy than weak 
swimmers. In any case, the energy spent is much smaller than if they had to travel the same distance on their own means. The differences between retention rates calculated in the present study and the values reported in Helfrich and Pineda (2003) are due both to features of the velocity fields that are absent in the two dimensional simulation, and to the different behavior of the particles. In HP, the particles are constantly swimming up. As a result, the only place where particles can accumulate is along stagnation points. Regions of closed streamlines (which were present in the 2D simulation) cannot accumulate particles, since particles will eventually escape. However, if particles swim up only in response to downelling currents, it is possible for them to cross into regions of closed streamline and remain inside.

In summary, our findings show that the biological response of swimming upward when exposed to downwelling currents enables plankton to accumulate in realistically simulated fronts and to be transported in the Lagrangian sense over considerable distances.

Acknowledgments. This study was supported through the NSF Biocomplexity Program, grant 000-83-976. Alberto Scotti completed part of the work while at Arizona State University as a Visiting Professor and would like to thank Prof. J. Fernando for his gracious hospitality. Calculations were performed on hardware maintained by ITS on the UNC-CH campus. The authors wish to thank Dr. Franks and two anonymous referees for their constructive criticism.

\section{APPENDIX}

During the two-dimensional setup of the experiment, Kelvin-Helmholtz billows regularly developed downstream of the head and grew to considerable size. When allowed to become three-dimensional, after a transient period, the production of Kelvin-Helmholtz billows came to a halt. The primary instability switched to spanwise fluctuations in the isopycnal surfaces associated to overturning instabilities in the warm side of the head (Fig. 18(a)).

To achieve a better insight into the basic structure of the flow we ran simulations at lower Grashof numbers. The reduced level of turbulence results in a "cleaner" view of the basic instability. Also, by using the same computational grid, we gain confidence that we are not just seeing the effects of numerical underresolution. Figure 19 shows the structure of the head at $G r=2.5 \times 10^{7}$. The spanwise-averaged density field shows that the stratification within the head is unstable, with a layer of heavier fluid sandwiched between lighter fluid above and below it, the latter being subdued from the frontal region. The light fluid supplying the head splits upstream of the head. Part flows along the surface, while some is diverted downward, mixes with heavy fluid and flows above the light fluid approaching from the frontal area. The resulting unstable configuration triggers a convective instability which separates the flow in three jets. At higher values of the Grashof number, the convective instability becomes more fragmented, but qualitatively the flow remains similar. To test whether this unexpected behavior was caused by the way in which we forced the flow to become three-dimensional, we repeated the experiment at $G r=10^{8}$, only this time instead of choosing a perturbed initial condition we started with a $2 \mathrm{D}$ initial condition, but added 

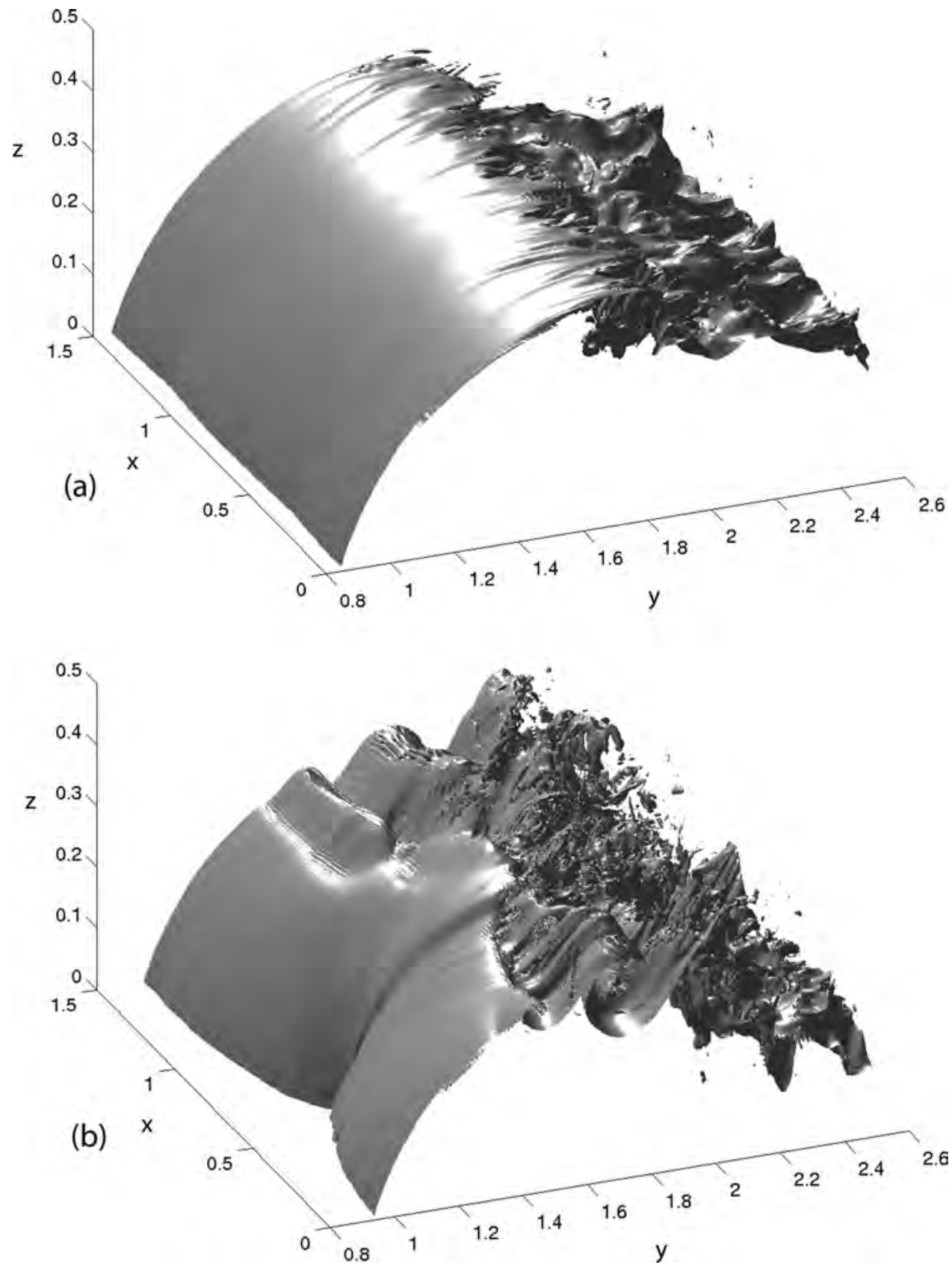

Figure 18. Isopycnal surface $\rho=0.1$ when Kelvin-Helmholtz billows are absent (a) and present (b). To facilitate the view, we have inverted the vertical axis. Note the spanwise fluctuations just upstream of the point where mixing begins in (a), and the cusp along the front in (b). 


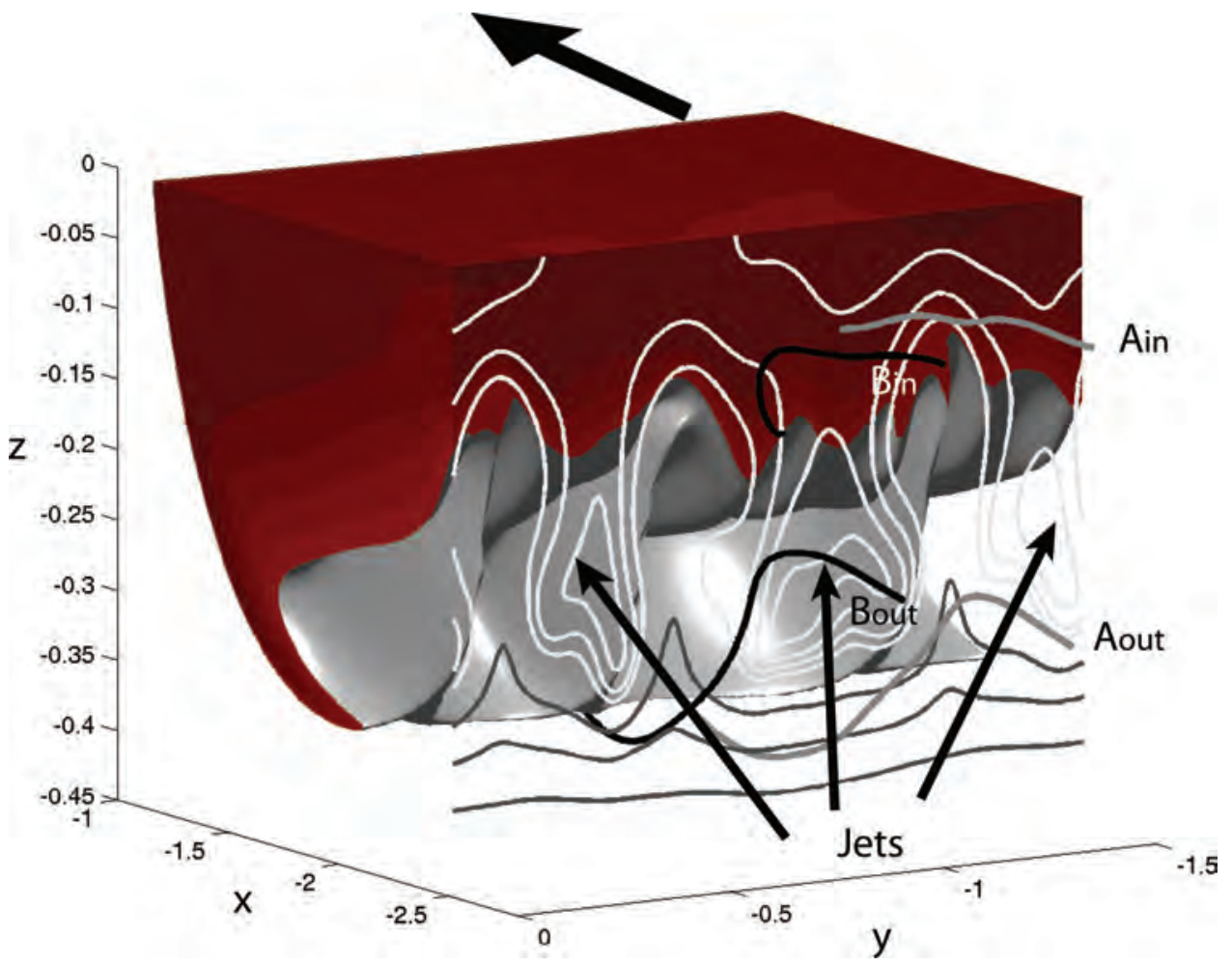

Figure 19. Structure of the head at low $G r$. The head is seen from behind, propagating in the positive $\mathrm{x}$ direction (arrow). The picture shows the volume occupied by unmixed warm fluid in shades of red. On the upstream side streamwise velocity isolines show how the flow splits in three jets, marked by arrows. Light (dark) grey lines denote streamwise velocity flowing into (away from) the head. Also plotted are the trajectories of two passive tracers initially released just below the surface at $\mathrm{z}=-0.05\left(\mathrm{~A}_{\mathrm{in}}\right.$ and $\mathrm{B}_{\mathrm{in}}$. The first $(\mathrm{A})$ keeps moving near the surface, until it reaches the front of the head, before being subdued. The other (B) is subdued shortly after being released, visualizing the flow responsible for the convective instability. As shown by the streamwise velocity isolines, the fastest forward flow is found in the core of the three jets, and not along the surface.

white noise to the velocity field at the upstream inlet. By the time the incoming fluid reaches the front of the gravity current, nonlinear interactions turn the artificial noise into "real" turbulence (Scotti and Meneveau, 1999). The amplitude of the noise was set so that the rms intensity of the resulting turbulent fluctuations just upstream of the head was about $0.5 \%$ of the speed of propagation of the gravity current, corresponding to the value reported in the experiments of Britter and Simpson (1978). In this case (Fig. 18(b)), we observed the development of Kelvin-Helmholtz billows. In fact, by turning on and off the noise at the inlet, it was possible to switch between the state with K-H billows and the state without.

We speculate that the linear instability of the basic flow is comprised of standing and traveling instabilities. The latter are the standard Kelvin-Helmholtz billows, and are likely 
to be the ones with the largest growth rate. The former, which a quick analysis of the basic flow shows to be centrifugal instabilities a la Taylor, are likely to posses a stronger initial amplitude under conditions of low or absent upstream noise, and generate a strong integrated disturbance that inhibits the formation of $\mathrm{K}-\mathrm{H}$ billows. This scenario has been observed experimentally and numerically in other flows (see e.g. White and Saric, 2005).

\section{REFERENCES}

Benjamin, T. B. 1968. Gravity currents and related phenomena. J. Fluid Mech., 31, 209-248.

Britter, R. E. and J. E. Simpson. 1978. Experiments on the dynamics of a gravity current head. J. Fluid Mech., 88, 223-240.

Cooper, A. L., R. P. Mied and G. J. Lindemann. 2001. Evolution of freely propagaing, two-dimensional surface gravity current fronts. J. Geophys. Res., 106(C8), 16887-16901.

Ewing, G. C. 1954. Slicks, surface films and internal waves. J. Mar. Res., 9, 161-187.

Franks, P. J .S. 1997. Spatial patterns in dense algal blooms. Limnol. Oceanogr., 42, 1297-1305.

2001. Turbulence avoidance: an alternate explanation of turbulence-enhanced ingestion rates in the field. Limnol. Oceanogr., 46, 959-963.

Härtel, C., E. Meiburg and F. Necker. 2000a. Analysis and direct numerical simulation of the flow at a gravity-current head. Part 1. Flow topology and front speed for slip and no-slip boundaries. J. Fluid Mech., 418, 189-212.

2000b. Analysis and direct numerical simulation of the flow at a gravity-current head. Part 2. The lobe-and-cleft instability. J. Fluid Mech., 418, 213-229.

Helfrich, K. R. and J. Pineda. 2003. Accumulation of particles in propagating fronts. Limnol. Oceanogr., 48, 1509-1520.

Hetland, R. D., D. J. McGillicuddy and R. S. Signell. 2003. Cross-frontal entrainment of plankton into a buoyant plume: the frog in tongue mechanism. J. Mar. Res., 60, 763-777.

Hosegood, P. and H. van Haren. 2004. Near-bed solibores over the continental slope in the FaeroeShetland Channel. Deep-Sea Res. II, 51, 2943-2971.

Jackson, C. R. 2004. An Atlas of Internal Solitary-like Internal Waves, Global Ocean Associates.

Klymak, J. M. and J. N. Moum. 2003. Internal solitary waves of elevation advancing on a shoaling shelf. Geophys. Res. Lett., 30, 2045. doi:10.1029/2003GL017706.

Lamb, K. 1997. Particle transport by non-breaking, solitary internal waves. J. Geophys. Res., $102,18641$.

Leichter, J. J., H. L. Stewart and S. L. Miller. 2003. Episodic nutrient transport of Florida coral reefs. Limnol. Oceanogr., 48, 1394-1407.

Lennert-Cody, C. E. and P. J. S. Franks. 1999. Plankton patchiness in high-frequency internal waves. MEPS, 186, 59-66.

Lowe, R. J., P. F. Linden and J. W. Rottman. 2002. A laboratory study of the velocity structure in an intrusive gravity current. J. Fluid Mech., 456, 33-48.

Manasseh, R., C.-Y. Ching and H. J. S. Fernando. 1998. The transition from density driven to wavedominated isolated flows. J. Fluid Mech., 361, 253-274.

Moin, P. and K. Manesh. 1998. Direct numerical simulation: A tool in turbulence research. Ann. Rev. Fluid Mech., 30, 539-578.

Mullin, M. M. 1993. Webs and scales. Physical and ecological processes in marine fish recruitment, University of Washington Press.

Norris, K. S. 1963. The function of temperature in the ecology of the percoid fish Girella nigricans (Ayres). Ecol. Monogr., 33, 23-62.

Orlanski, I. 1976. Simple boundary-condition for unbounded hyperbolic flows. J. Comp. Phys., $\underline{21,251-269 .}$ 
Ottino, J. M. 2004. The Kinematics of Mixing: Stretching, Chaos and Tranport, Cambridge University Press, $378 \mathrm{pp}$.

Özgökmen, T. M., P. E. Fischer, J. Duan and T. Iliescu. 2004. Three-dimensional turbulent bottom density currents from a high-order nonhydrostatic spectral element model. J. Phys. Oceanogr., 34, 2006-2026.

Pineda, J. 1991. Predictable upwelling and the shore-ward transport of planktonic larvae by internal tidal bores. Science, 253,548 .

1999. Circulation and larval distribution in internal tidal bore warm fronts. Limnol. Oceanogr. $44,1400-1414$.

Scotti, A. 2007. A numerical study of gravity currents propagating on a free-slip boundary. J. Fluid Mech. (Submitted).

Scotti, A. and C. Meneveau. 1999. A fractal model for large eddy simulation of turbulent flow. Physica D, 127, 198-232.

Scotti, A. and J. Pineda. 2004. Observation of very large and steep waves of elevation near the Massachusetts coast. Geophys. Res. Lett., 31, L22307.

Shanks, A. L. 1982. Onshore migration of megalopa by internal waves. Am. Zoologist, $22,902$. 1983. Surface slicks associated with tidally forced internal waves may transport pelagic larvae of benthic invertebrates and fish shoreward. MEPS, 13, 311-315.

1985. Behavioral basis of internal-wave-induced shore-ward transport of megalopae of the crab pachygrapsus crassipes. Mar. Ecol. Prog. Ser., 24, 289.

1987. The onshore transport of an oil spill by internal waves. Science, $235,1198$.

1988. Further support for the hypothesis that internal waves can cause shore-ward transport of larval invertebrates. Fisheries Bull., 86, 703.

Shanks, A. L. and W. G. Wright. 1987. Internal-wave-mediated shore-ward transport of cyprids, megalopae, and gammarids and correlated long-shore differences in the settling rate of intertidal barnacles. J. Exp. Mari. Biol. Ecol., 114, 1.

Simpson, J. E. 1999. Gravity Currents, in the Environment and the Laboratory. Cambridge University Press, $244 \mathrm{pp}$.

White, E. B. and W. S. Saric. 2005. Secondary instabilities of crossflow vortices. J. Fluid Mech., $525,275-308$.

Received: 6 June, 2006; revised: 2 November, 2006. 